\title{
DISCRETE TENOR MODELS FOR CREDIT RISKY PORTFOLIOS DRIVEN BY TIME-INHOMOGENEOUS LÉVY PROCESSES
}

\author{
ERNST EBERLEIN, ZORANA GRBAC, AND THORSTEN SCHMIDT
}

\begin{abstract}
The goal of this paper is to specify dynamic term structure models with discrete tenor structure for credit portfolios in a top-down setting driven by time-inhomogeneous Lévy processes. We provide a new framework, conditions for absence of arbitrage, explicit examples, an affine setup which includes contagion and pricing formulas for STCDOs and options on STCDOs. A calibration to iTraxx data with an extended Kalman filter shows an excellent fit over the full observation period. The calibration is done on a set of CDO tranche spreads ranging across six tranches and three maturities.
\end{abstract}

\section{INTRODUCTION}

Contrary to the single-obligor credit risk models, portfolio credit risk models consider a pool of credits consisting of different obligors and the adequate quantification of risk for the whole portfolio becomes a challenge. A good model for portfolio credit risk should incorporate two components: default risk, which includes in particular the dependence structure in the portfolio (also termed default correlation), and spread risk, which represents the risk related to changes of interest rates and changes in the credit quality of the obligors.

The main application of such a portfolio model which we discuss in Section 8 is the valuation of tranches of collateralized debt obligations (CDOs) and related derivatives. We would like to emphasize that variants of this model can be used for the valuation of other asset-backed securities. Currently, due to the sovereign credit crisis that has affected Europe, the issuance of so-called European Safe Bonds (ESBs) is discussed, where the underlying portfolio would consist of sovereign bonds of EU member states with fixed weights set by a strict rule which is proportional to GDP. Our model is easily adapted for pricing of such and other similar asset-backed securities whatever the precise specification of these instruments would be.

Generally speaking, CDOs are structured asset-backed securities, whose value and payments depend on a pool of underlying assets - such as bonds or loans - called the collateral. They consist of different tranches representing different risk classes, ranging from senior tranches with the lowest risk, over mezzanine tranches, to the equity tranche which carries the highest risk. If defaults occur in the collateral, the corresponding losses are transferred to investors in order of seniority, starting with the equity tranche.

Date: October 10, 2018.

Key words and phrases. collateralized debt obligations, loss process, single tranche CDO, ESB, top-down model, discrete tenor, market model, time-inhomogeneous Lévy processes, Libor rate, affine processes, extended Kalman filter, iTraxx.

The research of Z.G. benefited from the support of the Chaire Risque de Crédit, Fédération Bancaire Française and the DFG Research Center MATHEON. We would like to thank the associate editor and two anonymous referees for their valuable remarks. 
Among various portfolio credit risk models, there are two main approaches to be distinguished: the bottom-up approach where the default event of each individual obligor is modeled, and the top-down approach where the aggregate loss process of a given portfolio is modeled and the individual obligors in the portfolio are not identified. For a detailed overview of bottom-up and top-down approaches we refer to Lipton and Rennie (2011) and Bielecki, Crépey, and Jeanblanc (2010). The latter approach was investigated in a series of recent papers, among which we mention Schönbucher (2005), Sidenius, Piterbarg, and Andersen (2008), Ehlers and Schönbucher (2006, 2009), Arnsdorf and Halperin (2008), Longstaff and Rajan (2008), Errais, Giesecke, and Goldberg (2010), Filipović, Overbeck, and Schmidt (2011) and Cont and Minca (2013).

In this paper we present a dynamic term structure model with discrete tenor structure which studies portfolio credit risk in a top-down setting. The framework is developed in the spirit of the so-called Libor market model. The need for such an approach is illustrated in Carpentier (2009), and to our knowledge only Bennani and Dahan (2004) studied such models for CDOs. As in Filipović, Overbeck, and Schmidt (2011) we utilize $(T, x)$-bonds. In that paper a dynamic Heath-JarrowMorton (HJM) forward spread model for $(T, x)$-bonds has been analyzed under the assumption that $(T, x)$-bonds are traded for all maturities $T \in\left[0, T^{*}\right]$. Here we acknowledge the fact, that the set of traded maturities is only finite. This has important consequences for modeling and we introduce a new framework which takes this fact into account. We show that this framework possesses some clear advantages.

The first major difference is due to the fact that in the no-arbitrage condition in Theorem 5.2 one has to consider only finitely many maturities $T_{k}$. The HJMapproach instead has to guarantee the validity of this condition for a continuum of maturities. This restricts the model in an unnecessary way since traded products are only available for a small number of maturities. As we will show in the examples in section 6 one gains considerable additional freedom in the specification of arbitragefree models. See in particular Remark 5.3. The second difference is that we are able to include a contagion effect in an affine specification of this approach. It is evident that contagion is an important issue in the current crises. It should be mentioned that a model with only finitely many maturities can be extracted from the HJM framework, see Schmidt and Zabczyk (2012), which of course inherits the HJM-properties.

As driving processes for the dynamics of credit spreads, a wide class of timeinhomogeneous Lévy processes is used. This allows for jumps in the spread dynamics which are not only triggered by defaults in the underlying portfolio. In fact the empirical study in Cont and Kan (2011) reveals that jumps in the spread dynamics do not only occur at the default dates of the obligors in the portfolio, but they can also be caused by a macroeconomic event which is external to the portfolio. In Cont and Kan (2011) the bankruptcy of Lehman Brothers is given as an example of such an event. This is a weak point of some of the recently proposed portfolio credit risk models in which jumps in the spread dynamics occur only at default dates in the underlying portfolio (see a detailed discussion in Cont and Kan (2011)). In the model developed in the sequel we incorporate both types of jumps in the spread dynamics.

The model is calibrated to iTraxx data from January 2008 to August 2010 applying an extended Kalman filter to a two-factor affine diffusion specification of our approach, as proposed in Eksi and Filipović (2012). Contrary to the usual calibration to data from one day (see Cont, Deguest, and Kan (2010) for an overview and 
excellent empirical comparison), we calibrate the model to a much larger dataset running over three years. Already in the simple two-factor diffusion case a very good performance across different tranches and maturities is achieved.

The paper is structured as follows. In Section 2 we introduce the setting and basic notions. In Section 3 we describe the aggregate CDO loss process $L$ and the driving process $X$ and specify the dynamics of the credit spreads. Section 4 reviews the forward martingale measure approach. Section 5 contains the main results on the absence of arbitrage and Section 6 examines these results in a series of explicit examples. In Section 7 we focus attention on an affine specification which is able to incorporate contagion effects. In Section 8 we show how the valuation of derivatives can be facilitated by introducing appropriate defaultable forward measures and present a valuation formula for a single tranche CDO, which is the standard instrument for investing in a CDO. Moreover, we study the valuation of call options on STCDOs. Finally, in Section 9 we propose a two-factor affine specification and calibrate it to data from the iTraxx series.

\section{BASIC NOTIONS AND DEFINITIONS}

Let $T^{*}>0$ be a fixed time horizon and let a complete stochastic basis $\left(\Omega, \mathcal{G}, \mathbb{G}, \mathbb{Q}_{T^{*}}\right)$ be given, where $\mathcal{G}=\mathcal{G}_{T^{*}}$ and $\mathbb{G}=\left(\mathcal{G}_{t}\right)_{0 \leq t \leq T^{*}}$ is some filtration satisfying the usual conditions. For simplicity we write $\mathbb{Q}^{*}$ for $\mathbb{Q}_{T^{*}}$. The expectation with respect to $\mathbb{Q}^{*}$ is denoted by $\mathbb{E}^{*}$. The filtration $\mathbb{G}$ represents the filtration which contains all the information available in the market. All the price and interest rate processes in the sequel are adapted to it. Furthermore, assume that the tenor structure $0=T_{0}<T_{1}<\ldots<T_{n}=T^{*}$ is given. Set $\delta_{k}:=T_{k+1}-T_{k}$, for $k=0, \ldots, n-1$.

We assume that default-free zero coupon bonds with maturities $T_{1}, \ldots, T_{n}$ are traded in the market and denote by $P\left(t, T_{k}\right)$ the time- $t$ price of a default-free zero coupon bond with maturity $T_{k}$. For default-free zero coupon bonds $P\left(T_{k}, T_{k}\right)=1$ for all $k$. Furthermore we assume that $P\left(t, T_{k}\right)>0$ for any $0 \leq t \leq T_{k}$ and all $k$.

Furthermore, there is a pool of credit risky assets and we denote by $L=\left(L_{t}\right)_{t \geq 0}$ the nondecreasing aggregate loss process. Assume that the total nominal is normalized to 1 and denote by $\mathcal{I}:=[0,1]$ the set of loss fractions such that $L$ takes values in $\mathcal{I}$.

Remark 2.1. This approach is called top-down as we model the aggregate loss process directly. In the bottom-up approach one models instead the individual default times: for this, denote by $\tau_{1}, \ldots, \tau_{m}$ the default times of the credit risky securities in the collateral and their (possibly random) loss given default by $q_{1}, \ldots, q_{m}$. Then

$$
L_{t}=\sum_{i=1}^{m} q_{i} \mathbf{1}_{\left\{\tau_{i} \leq t\right\}} .
$$

Remark 2.2. The filtration $\mathbb{G}$ denotes the full market filtration to which the aggregate loss process is adapted. In Ehlers and Schönbucher (2009) the full market filtration is constructed as a progressive enlargement of a default-free filtration (known as a background or a reference filtration) with the default times in the portfolio under a certain version of the immersion hypothesis. Note that here $\mathbb{G}$ is general and we do not restrict ourselves to the case studied in Ehlers and Schönbucher (2009), In particular, the immersion hypothesis is not needed. 
Definition 2.3. A security which pays $1_{\left\{L_{T_{k}} \leq x\right\}}$ at $T_{k}$ is called $\left(T_{k}, x\right)$-bond. Its price at time $t \leq T_{k}$ is denoted by $P\left(t, T_{k}, x\right)$. Note that $P\left(t, T_{k}, x\right)=0$ on the set $\left\{L_{t}>x\right\}$.

If the market is free of arbitrage, $P\left(t, T_{k}, x\right)$ is nondecreasing in $x$ and

$$
P\left(t, T_{k}, 1\right)=P\left(t, T_{k}\right) .
$$

In Filipović, Overbeck, and Schmidt (2011) a forward rate model for (T, $x$ )-bonds has been analyzed under the assumption that $(T, x)$-bonds are traded for all maturities $T \in\left[0, T^{*}\right]$. Here we acknowledge the fact, that in practice the set of maturities for which the bonds are traded is finite.

Definition 2.4. The $\left(T_{k}, x\right)$-forward price is given by

$$
F\left(t, T_{k}, x\right):=\frac{P\left(t, T_{k}, x\right)}{P\left(t, T_{k}\right)}
$$

for $0 \leq t \leq T_{k}$.

The $\left(T_{k}, x\right)$-forward prices actually give the distribution of $L_{T_{k}}$ under the $\mathbb{Q}_{T_{k}}$ forward measure which will be defined later in (12). Indeed, note that if we take $P\left(\cdot, T_{k}\right)$ as the numeraire we obtain

$$
\begin{aligned}
\mathbb{Q}_{T_{k}}\left(L_{T_{k}} \leq x \mid \mathcal{G}_{t}\right) & =\frac{1}{P\left(t, T_{k}\right)} P\left(t, T_{k}\right) \mathbb{E}_{\mathbb{Q}_{T_{k}}}\left(\mathbf{1}_{\left\{L_{T_{k}} \leq x\right\}} \mid \mathcal{G}_{t}\right) \\
& =\frac{P\left(t, T_{k}, x\right)}{P\left(t, T_{k}\right)}=F\left(t, T_{k}, x\right) .
\end{aligned}
$$

Furthermore, we set for $k \in\{0, \ldots, n-1\}$ and $t \leq T_{k}$, on $\left\{L_{t} \leq x\right\}$,

$$
H\left(t, T_{k}, x\right):=\frac{F\left(t, T_{k+1}, x\right)}{F\left(t, T_{k}, x\right)} .
$$

This quantity relates to credit spreads as follows: intuitively, the credit spread quantifies the additional yield above the risk-free rate which the holder of a $\left(T_{k}, x\right)$-bond receives in compensation for taking the risk that $L$ jumps over the level $x$. Recall that for the classical Libor rate, with $\delta_{k}=T_{k+1}-T_{k}$,

$$
1+\delta_{k} \cdot \operatorname{LIBOR}\left(t, T_{k}\right)=\frac{P\left(t, T_{k}\right)}{P\left(t, T_{k+1}\right)} .
$$

If the credit spread is denoted by $c s\left(t, T_{k}, x\right)$, then on $\left\{L_{t} \leq x\right\}$

$$
\left(1+\delta_{k} c s\left(t, T_{k}, x\right)\right)\left(1+\delta_{k} \operatorname{LIBOR}\left(t, T_{k}\right)\right)=\frac{P\left(t, T_{k}, x\right)}{P\left(t, T_{k+1}, x\right)},
$$

and

$$
H\left(t, T_{k}, x\right)^{-1}=1+\delta_{k} c s\left(t, T_{k}, x\right)=\frac{P\left(t, T_{k}, x\right)}{P\left(t, T_{k+1}, x\right)} \frac{P\left(t, T_{k+1}\right)}{P\left(t, T_{k}\right)} .
$$

As we shall see in Section 8, the quantities $H\left(t, T_{k}, x\right)$ and not the credit spreads $c s\left(t, T_{k}, x\right)$ appear as the main ingredients in pricing formulas for portfolio credit derivatives. 
By induction we obtain the following decomposition of the $\left(T_{k}, x\right)$-forward price. For $t \in\left[0, T^{*}\right]$, let $j(t):=\inf \left\{i \in \mathbb{N}: T_{i-1}<t \leq T_{i}\right\}$, with the convention $j(0)=0$, denote the unique integer $j$ such that $T_{j-1}<t \leq T_{j}$. From (3) we obtain

$$
F\left(t, T_{k}, x\right)=\mathbf{1}_{\left\{L_{t} \leq x\right\}} F\left(t, T_{j(t)}, x\right) \prod_{i=j(t)}^{k-1} H\left(t, T_{i}, x\right) .
$$

Summarizing, the model has three ingredients to be specified: the dynamics of the loss process $L$, the credit spread via $H$ and the $F\left(t, T_{j(t)}, x\right)$. This of course should be done in a way which excludes arbitrage and leads to tractable pricing formulas. Both points will be discussed in the next sections.

\section{INGREDIENTS OF THE MODEL}

Let us now describe the processes which drive the model. A realistic assumption is that the dynamics of defaultable quantities related to the assets in the given portfolio is influenced by the aggregate loss process $L$. This means that when a default occurs in the portfolio, the default intensities of the other assets may be affected as well. In order to incorporate these features, we design a model where two sources of randomness appear:

(1) a time-inhomogeneous Lévy process $X$ representing the market randomness, which is driving the default-free and the pre-default dynamics

(2) the aggregate loss process $L$ for the given pool of credits.

From now on we assume that these two processes are independent with càdlàg trajectories. Note that this implies that there are no simultaneous jumps of $X$ and $L$. The independence assumption can be relaxed at the cost of having less explicit expressions. However, joint jumps in credit spreads and the loss process are incorporated via an explicit contagion mechanism, see (11).

The definition and main properties of time-inhomogeneous Lévy processes can be found for example in Eberlein and Kluge (2006). We recall that these processes are also known as processes with independent increments and absolutely continuous characteristics (PIIAC, cf. Jacod and Shiryaev (2003)), or additive processes in the sense of Sato (1999). For general semimartingale theory we refer to the book by Jacod and Shiryaev (2003), whose notation we adopt throughout the paper. Timeinhomogeneous Lévy processes have already been used in term structure modeling of interest rates because of their analytical tractability combined with a high degree of flexibility, which allows for an adequate fit of the term structure of volatility smiles, i.e. of the change of the smile across maturities; see Eberlein and Kluge (2006) and Eberlein and Koval (2006). In credit risk modeling there is also evidence that processes with jumps are a convenient choice as driving processes for the dynamics of credit spreads; see Cont and Kan (2011, p. 118), where the observation that the jumps in the spreads are not only tied to defaults in the underlying portfolio is stated.

Before giving a precise characterization of the driving process, let us describe the aggregate loss process $L$ in more detail. We assume that $L_{t}=\sum_{s<t} \Delta L_{s}$ is an $\mathcal{I}$-valued nondecreasing marked point process with absolutely continuous $\mathbb{Q}^{*}$ compensator

$$
\nu^{L}(\mathrm{~d} t, \mathrm{~d} y)=F_{t}^{L}(\mathrm{~d} y) \mathrm{d} t,
$$


where $F^{L}$ is a transition kernel from $\left(\Omega \times\left[0, T^{*}\right], \mathcal{P}\right)$ into $(\mathbb{R}, \mathcal{B}(\mathbb{R}))$ and $\mathcal{P}$ denotes the predictable $\sigma$-algebra on $\Omega \times\left[0, T^{*}\right]$.

Note that $L$ is a semimartingale with finite variation and with canonical representation

$$
L=x * \mu^{L}=x *\left(\mu^{L}-\nu^{L}\right)+x * \nu^{L},
$$

where $\mu^{L}$ denotes its random measure of jumps. Moreover, $L$ is a special semimartingale since its jumps are bounded by 1 .

The indicator process $\mathbf{1}_{\left\{L_{t} \leq x\right\}}$ is a càdlàg, decreasing process with intensity process

$$
\lambda(t, x)=F_{t}^{L}\left(\left(x-L_{t}, 1\right] \cap \mathcal{I}\right) ;
$$

i.e. the process

$$
M_{t}^{x}=\mathbf{1}_{\left\{L_{t} \leq x\right\}}+\int_{0}^{t} \mathbf{1}_{\left\{L_{s} \leq x\right\}} \lambda(s, x) \mathrm{d} s
$$

is a $\mathbb{Q}^{*}$-martingale (see Filipović, Overbeck, and Schmidt (2011), Lemma 3.1).

Let us provide an example for the loss process $L$. Note that the process defined in Remark 2.1 is also an example for $L$.

Example 3.1. Consider a compound Poisson process $Z=\left(Z_{t}\right)_{t \geq 0}$ with only positive jumps, defined as follows

$$
Z_{t}=\sum_{i=1}^{N_{t}} Y_{i}, \quad Z_{0}=0
$$

where $N=\left(N_{t}\right)_{t \geq 0}$ is a Poisson process with intensity $c$, and $Y_{i}, i \in \mathbb{N}$, are mutually independent, identically distributed random variables, independent of $N$, with distribution $P^{Y}$ on $\mathbb{R}^{+}$(e.g. take $P^{Y}$ to be a Gamma or an exponential distribution). The Lévy measure of $Z$ is given by $F^{Z}=c P^{Y}$. Next, we define the process $L=\left(L_{t}\right)_{t \geq 0}$ by

$$
L_{t}:=f\left(Z_{t}\right) \text {, }
$$

where $f: \mathbb{R}^{+} \rightarrow[0,1]$ is given by $f(x)=1-e^{-x}$. Since $f$ is a nondecreasing function, $L$ is a nondecreasing process taking values in $[0,1]$. Moreover, it is a purejump process by definition. The jumps of $L$ are given by

$$
\Delta L_{t}=e^{-Z_{t-}} f\left(\Delta Z_{t}\right) .
$$

Hence, $F_{t}^{L}$ equals

$$
F_{t}^{L}(E)=\int_{\mathbb{R}^{+}} \mathbf{1}_{E}\left(e^{-Z_{t-}} f(x)\right) F^{Z}(\mathrm{~d} x)=\int_{\mathbb{R}^{+}} c \mathbf{1}_{E}\left(e^{-Z_{t-}} f(x)\right) P^{Y}(\mathrm{~d} x),
$$

for $E \in \mathcal{B}\left(\mathbb{R}^{+} \backslash\{0\}\right)$, which completes the example.

Let $X$ be an $\mathbb{R}^{d}$-valued time-inhomogeneous Lévy process on the stochastic basis $\left(\Omega, \mathcal{G}, \mathbb{G}, \mathbb{Q}^{*}\right)$ with $X_{0}=0$ a.s. and canonical representation given by

$$
X_{t}=W_{t}+\int_{0}^{t} \int_{\mathbb{R}^{d}} x(\mu-\nu)(\mathrm{d} s, \mathrm{~d} x)
$$

where $W$ is a $d$-dimensional standard Brownian motion with respect to $\mathbb{Q}^{*}, \mu$ is the random measure of jumps of $X$ and $\nu$ such that $\nu(\mathrm{d} t, \mathrm{~d} x)=F_{t}(\mathrm{~d} x) \mathrm{d} t$ is its $\mathbb{Q}^{*}$-compensator. To ensure the existence of representation 10$)$ we assume 
(A1) There exist constants $\tilde{C}, \varepsilon>0$ such that

$$
\sup _{0 \leq t \leq T^{*}}\left(\int_{|y|>1} \exp \langle u, y\rangle F_{t}(\mathrm{~d} y)\right)<\infty,
$$

for every $u \in[-(1+\varepsilon) \tilde{C},(1+\varepsilon) \tilde{C}]^{d}$.

This assumption entails the existence of exponential moments of $X$, i.e. $\mathbb{E}^{*}\left[\exp \left\langle u, X_{t}\right\rangle\right]<$ $\infty$, for all $t \in\left[0, T^{*}\right]$ and $u$ as above; cf. Lemma 6 in Eberlein and Kluge (2006).

The main ingredient for our model is the specification of the dynamics of the credit spreads via specification of $H$. We assume that

$$
\begin{aligned}
H\left(t, T_{k}, x\right) & =H\left(0, T_{k}, x\right) \exp \left(\int_{0}^{t} a\left(s, T_{k}, x\right) d s+\int_{0}^{t} b\left(s, T_{k}, x\right) d X_{s}\right. \\
& \left.+\int_{0}^{t} \int_{\mathcal{I}} c\left(s, T_{k}, x ; y\right) \mu^{L}(d s, d y)\right),
\end{aligned}
$$

where we impose the following assumptions $(\mathcal{O}$ and $\mathcal{P}$ denote respectively the optional and the predictable $\sigma$-algebra on $\left.\left(\Omega \times\left[0, T^{*}\right]\right)\right)$ :

(A2) For all $T_{k}$ there is an $\mathbb{R}_{+}^{d}$-valued process $b\left(s, T_{k}, x\right)$, which as a function of $(s, x) \mapsto b\left(s, T_{k}, x\right)$ is $\mathcal{P} \otimes \mathcal{B}(\mathcal{I})$-measurable. Moreover,

$$
\sup _{s \in\left[0, T^{*}\right], x \in \mathcal{I}, \omega \in \Omega} \sum_{k=1}^{n-1} b^{j}\left(s, T_{k}, x\right) \leq \tilde{C}
$$

for every coordinate $j \in\{1, \ldots, d\}$, where $\tilde{C}>0$ is the constant from (A1). If $s>T_{k}$, then $b\left(s, T_{k}, x\right)=0$.

(A3) For all $T_{k}$ there is an $\mathbb{R}$-valued process $c\left(s, T_{k}, x ; y\right)$, which is called the contagion parameter and which as a function of $(s, x, y) \mapsto c\left(s, T_{k}, x ; y\right)$ is $\mathcal{P} \otimes \mathcal{B}(\mathcal{I}) \otimes \mathcal{B}(\mathcal{I})$-measurable. We also assume

$$
\sup _{s \leq T_{k}, x, y \in \mathcal{I}, \omega \in \Omega}\left|c\left(s, T_{k}, x ; y\right)\right|<\infty
$$

and $c\left(s, T_{k}, x ; y\right)=0$ for $s>T_{k}$.

(A4) The initial term structure $P\left(0, T_{k}, x\right)$ is strictly positive, strictly decreasing in $k$ and satisfies

$$
F\left(0, T_{k}, x\right)=\frac{P\left(0, T_{k}, x\right)}{P\left(0, T_{k}\right)} \geq \frac{P\left(0, T_{k+1}, x\right)}{P\left(0, T_{k+1}\right)}=F\left(0, T_{k+1}, x\right) .
$$

The drift term $a\left(\cdot, T_{k}, \cdot\right)$, for every $T_{k}$, is an $\mathbb{R}$-valued, $\mathcal{O} \otimes \mathcal{B}(\mathcal{I})$-measurable process such that $a\left(s, T_{k}, x\right)=0$, for $s>T_{k}$, which will be specified later. Note that this together with assumptions (A2) and (A3) implies that $H\left(t, T_{i}, x\right)$ remains constant after $T_{i}$, i.e. $H\left(t, T_{i}, x\right)=H\left(T_{i}, T_{i}, x\right)$, for $t \geq T_{i}$.

Remark 3.2. Specifying the dynamics of $H$ in this way, we allow for two kinds of jumps: the jumps caused by market forces, represented by the time-inhomogeneous Lévy process $X$, and the jumps caused by defaults in the portfolio, represented through the aggregate loss process $L$, which allows for contagion effects. 


\section{THE FORWARD MEASURES}

In a short excursion we recall the most important results from default-free Libor models and introduce the forward martingale measures.

In default-free discrete tenor models the forward martingale measures are constructed by backward induction, together with the forward Libor rates. The measure $\mathbb{Q}^{*}=\mathbb{Q}_{T^{*}}=\mathbb{Q}_{T_{n}}$ plays the role of the forward measure associated with the settlement date $T_{n}$ and is called the terminal forward measure. We shall write $W^{T_{n}}$ for $W$ and $\nu^{T_{n}}$ for $\nu$ when we wish to emphasize that $\mathbb{Q}^{*}$ is the terminal forward measure.

The forward measure $\mathbb{Q}_{T_{k}}$ is defined on $\left(\Omega, \mathcal{G}_{T_{k}}\right)$ by its Radon-Nikodym derivative with respect to $\mathbb{Q}_{T_{n}}$, i.e.

$$
\left.\frac{\mathrm{d} \mathbb{Q}_{T_{k}}}{\mathrm{~d} \mathbb{Q}_{T_{n}}}\right|_{\mathcal{G}_{t}}=\frac{P\left(0, T_{n}\right)}{P\left(0, T_{k}\right)} \frac{P\left(t, T_{k}\right)}{P\left(t, T_{n}\right)} .
$$

We assume that this density has the following representation as a stochastic exponential:

$$
\left.\frac{\mathrm{d} \mathbb{Q}_{T_{k}}}{\mathrm{~d} \mathbb{Q}_{T_{n}}}\right|_{\mathcal{G}_{t}}=\mathcal{E}_{t}\left(\int_{0} \alpha\left(s, T_{k}\right) d W_{s}+\int_{0} \int_{\mathbb{R}^{d}}\left(\beta\left(s, T_{k}, y\right)-1\right)(\mu-\nu)(d s, d y)\right),
$$

where $\alpha \in L(W)$ and $\beta \in G_{\mathrm{loc}}(\mu)$ in the sense of Theorem III.7.23 in Jacod and Shiryaev (2003); for definitions of $L(W)$ and $G_{\text {loc }}(\mu)$ see the same textbook, page 207 and page 72 respectively. Then, applying Girsanov's theorem, we deduce that

$$
W_{t}^{T_{k}}:=W_{t}-\int_{0}^{t} \alpha\left(s, T_{k}\right) \mathrm{d} s
$$

is a $d$-dimensional standard Brownian motion with respect to $\mathbb{Q}_{T_{k}}$, and

$$
\nu^{T_{k}}(\mathrm{~d} s, \mathrm{~d} y):=\beta\left(s, T_{k}, y\right) \nu(\mathrm{d} s, \mathrm{~d} y)=F_{s}^{T_{k}}(\mathrm{~d} y) \mathrm{d} s,
$$

is the $\mathbb{Q}_{T_{k}}$-compensator of $\mu$, where $F_{s}^{T_{k}}(\mathrm{~d} y)=\beta\left(s, T_{k}, y\right) F_{s}(\mathrm{~d} y)$. See Eberlein and Özkan (2005), Section 4, pp. 338-342, for the detailed construction of Libor rates which are driven by a Lévy process.

We denote by $\nu^{L, T_{k}}(d t, d x)=F_{t}^{L, T_{k}}(d x) d t$ the $\mathbb{Q}_{T_{k}}$-compensator of the random measure $\mu^{L}$ of the jumps of the loss process. The existence of $F_{t}^{L, T_{k}}$ follows in the same way as the existence of $F_{t}^{T_{k}}$ in $(15)$.

Remark 4.1 (Constant term structure). If the price processes for default-free bonds $\left(P\left(t, T_{k}\right)\right)_{0 \leq t \leq T_{k}}$ are constant equal to 1 for every $k=1, \ldots, n$, all forward measures coincide, i.e.

$$
\mathbb{Q}_{T_{1}}=\cdots=\mathbb{Q}_{T_{n}}=\mathbb{Q}^{*}
$$

\section{Absence of arbitrage}

The goal of this section is to identify conditions which guarantee absence of arbitrage in our setting. It is well-known that the model is free of arbitrage if all $\left(T_{k}, x\right)$-bonds discounted with a suitable numeraire are local martingales and we choose default-free bonds as numeraires. 
The quantity $F\left(t, T_{j(t)}, x\right)$ given in (5) is the forward bond price for the closest maturity from time $t$ (typically less than 3 months). In the following discussion of absence of arbitrage we do not have to consider this particular forward bond price. The reason for this is that the market trades only financial instruments whose first tenor date (payment date) is at least a full tenor period away. As a consequence, we consider $P\left(\cdot, T_{k}, x\right)$ as traded assets, with $k \in\{2, \ldots, n\}$, and study the question if $\left(F\left(t, T_{k}, x\right)\right)_{0 \leq t \leq T_{k-1}}$ are $\mathbb{Q}_{T_{k}}$-local martingales for any $k \in\{2, \ldots, n\}$. The following lemma shows that the numeraires can be interchanged arbitrarily.

Lemma 5.1. There is equivalence between:

(a) For each $k=2, \ldots, n$ the process

$$
\left(F\left(t, T_{k}, x\right)\right)_{0 \leq t \leq T_{k-1}}
$$

is a $\mathbb{Q}_{T_{k}}$-local martingale.

(b) For each $k, i=2, \ldots, n$ the process

$$
\left(\frac{P\left(t, T_{k}, x\right)}{P\left(t, T_{i}\right)}\right)_{0 \leq t \leq T_{i} \wedge T_{k-1}}
$$

is a $\mathbb{Q}_{T_{i}}$-local martingale.

Proof: It suffices to note that for fixed $i, k \in\{2, \ldots, n\}$ such that $i \geq k$ (the other case is treated in the same way) we have

$$
\frac{P\left(t, T_{k}, x\right)}{P\left(t, T_{i}\right)}=F\left(t, T_{k}, x\right) \frac{P\left(t, T_{k}\right)}{P\left(t, T_{i}\right)}
$$

where $F\left(\cdot, T_{k}, x\right)=\frac{P\left(\cdot, T_{k}, x\right)}{P\left(\cdot, T_{k}\right)}$ is a $\mathbb{Q}_{T_{k}}$-local martingale by (a) and $\frac{P\left(\cdot, T_{k}\right)}{P\left(\cdot, T_{i}\right)}$ is the density process of the measure $\mathbb{Q}_{T_{k}}$ relative to $\mathbb{Q}_{T_{i}}$, up to a norming constant (cf. equation (12)). Then $\frac{P\left(\cdot, T_{k}, x\right)}{P\left(\cdot, T_{i}\right)}$ is a $\mathbb{Q}_{T_{i}}$-local martingale by Proposition III.3.8 in Jacod and Shiryaev (2003), The implication $(a) \Rightarrow(b)$ is thus shown. $(b) \Rightarrow(a)$ is obvious.

Now regarding the discussion at the beginning of this section, we specify (5) further as follows

$$
F\left(t, T_{k}, x\right):=\mathbf{1}_{\left\{L_{t} \leq x\right\}} \prod_{i=0}^{k-1} H\left(t, T_{i}, x\right),
$$

for any $0 \leq t \leq T_{k-1}$ with $H\left(t, T_{i}, x\right)$ given by 111$)$. Recall that $H\left(t, T_{i}, x\right)$ remains constant for $t>T_{i}$ by assumption. We examine conditions for absence of arbitrage, i.e. necessary and sufficient conditions for the $\left(T_{k}, x\right)$-forward price process $F\left(\cdot, T_{k}, x\right)$ being a local martingale under the forward measure $\mathbb{Q}_{T_{k}}$, for $k=2, \ldots, n$.

Set

$$
\begin{aligned}
D\left(t, T_{k}, x\right) & :=\sum_{i=1}^{k-1} a\left(t, T_{i}, x\right)+\frac{1}{2}\left\|\sum_{i=1}^{k-1} b\left(t, T_{i}, x\right)\right\|^{2} \\
& +\left\langle\sum_{i=1}^{k-1} b\left(t, T_{i}, x\right), \alpha\left(t, T_{k}\right)\right\rangle \\
& +\int_{\mathbb{R}^{d}}\left(e^{\left\langle\sum_{i=1}^{k-1} b\left(t, T_{i}, x\right), y\right\rangle}-1-\left\langle\sum_{i=1}^{k-1} b\left(t, T_{i}, x\right), y\right\rangle \beta\left(t, T_{k}, y\right)^{-1}\right) F_{t}^{T_{k}}(d y),
\end{aligned}
$$


where $\alpha$ and $\beta$ were introduced in $(13)$. Recall that $\nu^{L, T_{k}}(\mathrm{~d} t, \mathrm{~d} x)=F_{t}^{L, T_{k}}(d x) d t$ is the $\mathbb{Q}_{T_{k}}$-compensator of the random measure of jumps $\mu^{L}$. Analogously to (8), we get that

$$
M_{t}^{x, T_{k}}:=\mathbf{1}_{\left\{L_{t} \leq x\right\}}+\int_{0}^{t} \mathbf{1}_{\left\{L_{s} \leq x\right\}} \lambda^{T_{k}}(s, x) \mathrm{d} s
$$

is a $\mathbb{Q}_{T_{k}}$-martingale, where $\lambda^{T_{k}}(t, x):=F_{t}^{L, T_{k}}\left(\left(x-L_{t}, 1\right] \cap \mathcal{I}\right)$. By $\lambda^{1}$ we denote the Lebesgue measure on $\mathbb{R}$.

Theorem 5.2. Assume that (A1)-(A4) are in force and let $k \in\{2, \ldots, n\}, x \in \mathcal{I}$. Then the process $\left(F\left(t, T_{k}, x\right)\right)_{0 \leq t \leq T_{k-1}}$ given by (16) is a $\mathbb{Q}_{T_{k}}$-local martingale if and only if

$$
D\left(t, T_{k}, x\right)=\lambda^{T_{k}}(t, x)-\int_{\mathcal{I}}\left(e^{\sum_{i=1}^{k-1} c\left(t, T_{i}, x ; y\right)}-1\right) \mathbf{1}_{\left\{L_{t-}+y \leq x\right\}} F_{t}^{L, T_{k}}(d y)
$$

on the set $\left\{L_{t} \leq x\right\}, \lambda^{1} \otimes \mathbb{Q}_{T_{k}}$-a.s.

Remark 5.3. Note that in the HJM term structure models, by considering the continuum of maturities one puts unnecessary restrictions on the model. It is a major advantage of models with discrete tenor structure that only those maturities are considered which are traded in the market. It will become clear in the various examples, which are discussed in Section 6, that the drift condition 190 can be satisfied while there is still a high degree of freedom to specify the intensity of the loss process. This is not the case in the HJM framework, where the risky short rate is directly connected to the intensity of the loss process, see equation (3.11) in Filipović, Overbeck, and Schmidt (2011). For example, we are able to specify the dynamics of the spreads and still have an arbitrary intensity of the loss process. Moreover we are able to specify an affine version of the model which includes contagion.

Proof: We calculate first the dynamics of the forward price processes under the forward measures and then derive the drift conditions. We fix $x$ and $T_{k}$ and define

$$
G(t)=G(t, k, x):=\prod_{i=0}^{k-1} H\left(t, T_{i}, x\right)
$$

such that $F\left(t, T_{k}, x\right)=G(t) \mathbf{1}_{\left\{L_{t} \leq x\right\}}$. Using integration by parts yields

$$
\begin{aligned}
\mathrm{d} F\left(t, T_{k}, x\right) & =G(t-) \mathrm{d} \mathbf{1}_{\left\{L_{t} \leq x\right\}}+\mathbf{1}_{\left\{L_{t-} \leq x\right\}} \mathrm{d} G(t)+\mathrm{d}\left[G, \mathbf{1}_{\{L . \leq x\}}\right]_{t} \\
& =:\left(1^{\prime}\right)+\left(2^{\prime}\right)+\left(3^{\prime}\right) .
\end{aligned}
$$

We deal separately with each of the above three summands. Regarding $\left(1^{\prime}\right)$, equation (18) yields

$$
\begin{aligned}
\mathrm{d} \mathbf{1}_{\left\{L_{t} \leq x\right\}} & =\mathrm{d} M_{t}^{x, T_{k}}-\mathbf{1}_{\left\{L_{t} \leq x\right\}} \lambda^{T_{k}}(t, x) \mathrm{d} t \\
& =\mathbf{1}_{\left\{L_{t-} \leq x\right\}} \mathrm{d} M_{t}^{x, T_{k}}-\mathbf{1}_{\left\{L_{t-} \leq x\right\}} \lambda^{T_{k}}(t, x) \mathrm{d} t \\
& =\mathbf{1}_{\left\{L_{t-} \leq x\right\}}\left(\mathrm{d} M_{t}^{x, T_{k}}-\lambda^{T_{k}}(t, x) \mathrm{d} t\right),
\end{aligned}
$$


since a short computation shows that $\mathrm{d} M_{t}^{x, T_{k}}=\mathbf{1}_{\left\{L_{t-} \leq x\right\}} \mathrm{d} M_{t}^{x, T_{k}}$. Hence,

$$
\begin{aligned}
\left(1^{\prime}\right) & =G(t-) 1_{\left\{L_{t-} \leq x\right\}}\left(\mathrm{d} M_{t}^{x, T_{k}}-\lambda^{T_{k}}(t, x) \mathrm{d} t\right) \\
& =F\left(t-, T_{k}, x\right)\left(\mathrm{d} M_{t}^{x, T_{k}}-\lambda^{T_{k}}(t, x) \mathrm{d} t\right) .
\end{aligned}
$$

Regarding (2'), we obtain using (11)

$$
\begin{aligned}
G(t) & =G(0) \exp \left(\int_{0}^{t} \sum_{i=1}^{k-1} a\left(s, T_{i}, x\right) d s\right. \\
& \left.+\int_{0}^{t} \sum_{i=1}^{k-1} b\left(s, T_{i}, x\right) d X_{s}+\int_{0}^{t} \int_{\mathcal{I}}^{k-1} \sum_{i=1}^{k-1} c\left(s, T_{i}, x ; y\right) \mu^{L}(d s, d y)\right) .
\end{aligned}
$$

By Itô's formula for semimartingales

$$
\begin{aligned}
\left(2^{\prime}\right) & =F\left(t-, T_{k}, x\right)\left(\left(\sum_{i=1}^{k-1} a\left(t, T_{i}, x\right)+\frac{1}{2}\left\|\sum_{i=1}^{k-1} b\left(t, T_{i}, x\right)\right\|^{2}\right) d t\right. \\
& +\sum_{i=1}^{k-1} b\left(t, T_{i}, x\right) d W_{t}+\int_{\mathbb{R}^{d}}\left(e^{\left\langle\sum_{i=1}^{k-1} b\left(t, T_{i}, x\right), y\right\rangle}-1\right)(\mu-\nu)(d t, d y) \\
& +\int_{\mathbb{R}^{d}}\left(e^{\left\langle\sum_{i=1}^{k-1} b\left(t, T_{i}, x\right), y\right\rangle}-1-\left\langle\sum_{i=1}^{k-1} b\left(t, T_{i}, x\right), y\right\rangle\right) \nu(d t, d y) \\
& \left.+\int_{\mathcal{I}}\left(e^{\sum_{i=1}^{k-1} c\left(t, T_{i}, x ; y\right)}-1\right) \mu^{L}(d t, d y)\right) .
\end{aligned}
$$

We finally incorporate the dynamics of the driving processes under the $T_{k}$-forward measure and obtain by (14) and (15)

$$
\begin{aligned}
\left(2^{\prime}\right) & =F\left(t-, T_{k}, x\right)\left(\left(\sum_{i=1}^{k-1} a\left(t, T_{i}, x\right)+\frac{1}{2}\left\|\sum_{i=1}^{k-1} b\left(t, T_{i}, x\right)\right\|^{2}\right.\right. \\
& +\left\langle\sum_{i=1}^{k-1} b\left(t, T_{i}, x\right), \alpha\left(t, T_{k}\right)\right\rangle \\
& +\int_{\mathbb{R}^{d}}\left(e^{\left\langle\sum_{i=1}^{k-1} b\left(t, T_{i}, x\right), y\right\rangle}-1-\left\langle\sum_{i=1}^{k-1} b\left(t, T_{i}, x\right), y\right\rangle \beta\left(t, T_{k}, y\right)^{-1}\right) F_{t}^{T_{k}}(d y) \\
& \left.+\int_{\mathcal{I}}\left(e^{\sum_{i=1}^{k-1} c\left(t, T_{i}, x ; y\right)}-1\right) F_{t}^{L, T_{k}}(d y)\right) d t \\
& +\sum_{i=1}^{k-1} b\left(t, T_{i}, x\right) d W_{t}^{T_{k}}+\int_{\mathbb{R}^{d}}\left(e^{\sum_{i=1}^{k-1} b\left(t, T_{i}, x\right) y}-1\right)\left(\mu-\nu^{T_{k}}\right)(d t, d y) \\
& \left.+\int_{\mathcal{I}}\left(e^{\sum_{i=1}^{k-1} c\left(t, T_{i}, x ; y\right)}-1\right)\left(\mu^{L}-\nu^{L, T_{k}}\right)(\mathrm{d} t, d y)\right) .
\end{aligned}
$$


It remains to calculate the covariation part $\left(3^{\prime}\right)$. Since $\mathbf{1}_{\left\{L_{t} \leq x\right\}}$ does not have a continuous martingale part, we conclude

$$
\left[G, \mathbf{1}_{\{L . \leq x\}}\right]_{t}=\sum_{s \leq t} \Delta G(s) \Delta \mathbf{1}_{\left\{L_{s} \leq x\right\}} .
$$

Moreover,

$$
\begin{aligned}
\Delta \mathbf{1}_{\left\{L_{s} \leq x\right\}}(\omega) & =\mathbf{1}_{\left\{L_{s} \leq x\right\}}(\omega)-\mathbf{1}_{\left\{L_{s-} \leq x\right\}}(\omega) \\
& =\left\{\begin{aligned}
-1 ; & \text { if } L_{s-}(\omega) \leq x \text { and } L_{s}(\omega)>x \\
0 ; & \text { otherwise } .
\end{aligned}\right.
\end{aligned}
$$

Therefore,

$$
\Delta \mathbf{1}_{\left\{L_{s} \leq x\right\}}=-\mathbf{1}_{\left\{L_{s-} \leq x, L_{s}>x\right\}}=-\mathbf{1}_{\left\{L_{s-} \leq x, L_{s-}+\Delta L_{s}>x\right\}}
$$

and it follows

$$
\Delta \mathbf{1}_{\left\{L_{s} \leq x\right\}}=\int_{\mathbb{R}} z \mu^{\mathbf{1}_{\{L, \leq x\}}}(\{s\}, \mathrm{d} z)=-\int_{\mathcal{I}} \mathbf{1}_{\left\{L_{s-} \leq x\right\}} \mathbf{1}_{\left\{L_{s-}+y>x\right\}} \mu^{L}(\{s\}, \mathrm{d} y) .
$$

In (20) we already computed the dynamics of $G$ and hence, we deduce

$$
\left(3^{\prime}\right)=-G(t-) \mathbf{1}_{\left\{L_{t-} \leq x\right\}} \int_{\mathcal{I}}\left(e^{\sum_{i=1}^{k-1} c\left(t, T_{i}, x ; y\right)}-1\right) \mathbf{1}_{\left\{L_{t-}+y>x\right\}} \mu^{L}(\mathrm{~d} t, \mathrm{~d} y) .
$$

Summing up the calculations, we obtain on $\left\{F\left(t-, T_{k}, x\right)>0\right\}$

$$
\begin{aligned}
\frac{d F\left(t, T_{k}, x\right)}{F\left(t-, T_{k}, x\right)} & =\left(-\lambda^{T_{k}}(t, x)+D\left(t, T_{k}, x\right)\right. \\
& +\int_{\mathcal{I}}\left(e^{\sum_{i=1}^{k-1} c\left(t, T_{i}, x ; y\right)}-1\right) F_{t}^{L, T_{k}}(d y) \\
& \left.-\int_{\mathcal{I}}\left(e^{\sum_{i=1}^{k-1} c\left(t, T_{i}, x ; y\right)}-1\right) \mathbf{1}_{\left\{L_{t-}+y>x\right\}} F_{t}^{L, T_{k}}(d y)\right) d t+d \tilde{M}_{t},
\end{aligned}
$$

for some local martingale $\tilde{M}$ and with $D\left(t, T_{k}, x\right)$ given by (17). This concludes the proof.

Remark 5.4. If the driving process $X$ does not have a Brownian part $W$, cf. (10), then an inspection of the proof shows that the model is free of arbitrage if the drift condition 19 holds when the term $D\left(t, T_{k}, x\right)$ is replaced by

$$
\begin{aligned}
D\left(t, T_{k}, x\right) & =\sum_{i=1}^{k-1} a\left(t, T_{i}, x\right) \\
& +\int_{\mathbb{R}^{d}}\left(e^{\left\langle\sum_{i=1}^{k-1} b\left(t, T_{i}, x\right), y\right\rangle}-1-\left\langle\sum_{i=1}^{k-1} b\left(t, T_{i}, x\right), y\right\rangle \beta\left(t, T_{k}, y\right)^{-1}\right) F_{t}^{T_{k}}(d y) .
\end{aligned}
$$




\section{EXAMPLES}

Up to now we defined the basic ingredients for specifying models with discrete tenor structure which are free of arbitrage. Note that these models can be calibrated to any given initial term structure. However, for a given family of intensities $(\lambda(t, x))_{t \geq 0, x \in \mathcal{I}}$ the drift has to satisfy condition (19). We shall now discuss some simple examples which already show the high degree of flexibility. Let us repeat that this is not the case in the HJM framework developed in Filipović, Overbeck, and Schmidt (2011) since the risky short rate in fact determines the form of the compensator of the loss process, see equation (5.1) in Filipović, Overbeck, and Schmidt (2011).

We start with any initial term structure, represented by a family $H\left(0, T_{k}, x\right)$ for

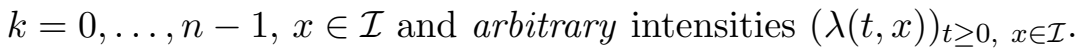

In the following examples we consider the case with constant term structure, see Remark 4.1. In this case the $T_{k}$-forward measures coincide and hence $\lambda^{T_{k}}(t, x)=$ $\lambda(t, x), \alpha\left(t, T_{k}\right)=0, \beta\left(t, T_{k}, y\right)=1, F_{t}^{T_{k}}(d y)=F_{t}(d y)$ and $F_{t}^{L, T_{k}}(d y)=F_{t}^{L}(d y)$.

Example 6.1 (Gaussian spread movements). This example will specify a simple $d$-factor Gaussian model. We consider no jumps in the spreads, i.e. $F_{t}(d y)=0$ and $c=0$ (no direct contagion). The volatilities $b\left(t, T_{i}, x\right)$ can be chosen arbitrarily, such that (A2) is satisfied. Thereafter we proceed iteratively:

(1) Let

$$
a\left(t, T_{1}, x\right)=\lambda(t, x)-\frac{1}{2}\left\|b\left(t, T_{1}, x\right)\right\|^{2} .
$$

(2) For $k=2, \ldots, n-1$ let

$$
a\left(t, T_{k}, x\right)=\frac{1}{2}\left(\left\|\sum_{i=1}^{k-1} b\left(t, T_{i}, x\right)\right\|^{2}-\left\|\sum_{i=1}^{k} b\left(t, T_{i}, x\right)\right\|^{2}\right) .
$$

Clearly, this model is free of arbitrage and can be calibrated to any given initial term structure. Note that the drift of the $H$ with closest maturity compensates the intensity $\lambda(t, x)$.

Example 6.2 (Lévy driven spread movements without Gaussian component). We assume pure-jump spread movements such that (21) holds. With $c=0$, we proceed analogously to the Gaussian example and start with arbitrary $F_{t}(d y)$ and $b\left(t, T_{i}, x\right)$ such that (A1) and (A2) are satisfied.

(1) Define

$$
a\left(t, T_{1}, x\right)=\lambda(t, x)-\int_{\mathbb{R}^{d}}\left(e^{\left\langle b\left(t, T_{1}, x\right), y\right\rangle}-1-\left\langle b\left(t, T_{1}, x\right), y\right\rangle\right) F_{t}(d y) .
$$

(2) For $k=2, \ldots, n-1$ define

$$
\begin{aligned}
a\left(t, T_{k}, x\right) & =\int_{\mathbb{R}^{d}}\left(e^{\left\langle\sum_{i=1}^{k-1} b\left(t, T_{i}, x\right), y\right\rangle}-1-\left\langle\sum_{i=1}^{k-1} b\left(t, T_{i}, x\right), y\right\rangle\right) F_{t}(d y) \\
& -\int_{\mathbb{R}^{d}}\left(e^{\left\langle\sum_{i=1}^{k} b\left(t, T_{i}, x\right), y\right\rangle}-1-\left\langle\sum_{i=1}^{k} b\left(t, T_{i}, x\right), y\right\rangle\right) F_{t}(d y) .
\end{aligned}
$$


Example 6.3 (Contagion). Next, we incorporate a direct contagion, i.e. $c$ does not vanish. We continue with the Lévy setting of Example 6.2. Contagion can be specified via the function $c$ : if the loss process has a jump of size $y$ at $t$, then

$$
H\left(t, T_{k}, x\right)=H\left(t-, T_{k}, x\right) e^{c\left(t, T_{k}, x ; y\right)}
$$

since $X$ and $L$ do not jump simultaneously. We can specify an arbitrage-free model with the following steps.

(1) Let

$$
\begin{aligned}
a\left(t, T_{1}, x\right) & =\lambda(t, x)-\int_{\mathbb{R}^{d}}\left(e^{\left\langle b\left(t, T_{1}, x\right), y\right\rangle}-1-\left\langle b\left(t, T_{1}, x\right), y\right\rangle\right) F_{t}(d y) \\
& -\int_{\mathcal{I}}\left(e^{c\left(t, T_{1}, x ; y\right)}-1\right) \mathbf{1}_{\left\{L_{t-}+y \leq x\right\}} F_{t}^{L}(d y) .
\end{aligned}
$$

(2) For $k=2, \ldots, n-1$ let

$$
\begin{aligned}
a\left(t, T_{k}, x\right) & =\int_{\mathbb{R}^{d}}\left(e^{\left\langle\sum_{i=1}^{k-1} b\left(t, T_{i}, x\right), y\right\rangle}-1-\left\langle\sum_{i=1}^{k-1} b\left(t, T_{i}, x\right), y\right\rangle\right) F_{t}(d y) \\
& +\int_{\mathcal{I}}\left(e^{\sum_{i=1}^{k-1} c\left(t, T_{1}, x ; y\right)}-1\right) \mathbf{1}_{\left\{L_{t-}+y \leq x\right\}} F_{t}^{L}(d y) \\
& -\int_{\mathbb{R}^{d}}\left(e^{\left\langle\sum_{i=1}^{k} b\left(t, T_{i}, x\right), y\right\rangle}-1-\left\langle\sum_{i=1}^{k} b\left(t, T_{i}, x\right), y\right\rangle\right) F_{t}(d y) \\
& -\int_{\mathcal{I}}\left(e^{\sum_{i=1}^{k} c\left(t, T_{i}, x ; y\right)}-1\right) \mathbf{1}_{\left\{L_{t-}+y \leq x\right\}} F_{t}^{L}(d y) .
\end{aligned}
$$

For some applications it may be interesting to simplify this setting further. As examples we discuss additive and multiplicative jumps in $H$.

(1) Additive jumps. We choose (deterministic) functions $C(t, x)$ and let

$$
e^{c\left(t, T_{k}, x ; y\right)}:=H\left(t-, T_{k}, x\right)^{-1} y C\left(T_{k}-t, x\right)+1 .
$$

This yields a jump of size $\Delta L_{t} C\left(T_{k}-t, x\right)$ of $H$ at time $t$, i.e.

$$
H\left(t, T_{k}, x\right)=H\left(t-, T_{k}, x\right)+\Delta L_{t} C\left(T_{k}-t, x\right),
$$

while the specification

$$
e^{c\left(t, T_{k}, x ; y\right)}:=\left(1+H\left(t-, T_{k}, x\right) y C\left(T_{k}-t, x\right)\right)^{-1}
$$

yields a jump of size $\delta_{k}^{-1} \Delta L_{t} C\left(T_{k}-t, x\right)$ in the credit spread as defined in formula (4):

$$
c s\left(t, T_{k}, x\right)=c s\left(t-, T_{k}, x\right)+\delta_{k}^{-1} \Delta L_{t} C\left(T_{k}-t, x\right) .
$$

(2) Multiplicative jumps. Again we choose (deterministic) functions $C(t, x)$ and let

$$
e^{c\left(t, T_{k}, x ; y\right)}:=y C\left(T_{k}-t, x\right) .
$$

In this case,

$$
H\left(t, T_{k}, x\right)=H\left(t-, T_{k}, x\right) \Delta L_{t} C\left(T_{k}-t, x\right)
$$


and in the drift condition we have the following simplification

$$
\begin{aligned}
\int_{\mathcal{I}}\left(e^{\sum_{i=1}^{k-1} c\left(t, T_{i}, x ; y\right)}-1\right) \mathbf{1}_{\left\{L_{t-}+y \leq x\right\}} F_{t}^{L}(d y) \\
=\int_{\mathcal{I}}\left(y^{k-1} \prod_{i=1}^{k-1} C\left(T_{i}-t, x\right)-1\right) \mathbf{1}_{\left\{L_{t-}+y \leq x\right\}} F_{t}^{L}(d y) .
\end{aligned}
$$

This expression depends on the distribution of the losses via $F_{t}^{L}$. For various approaches concerning the dependence on the loss process see Cont, Deguest, and Kan (2010).

Example 6.4 (Relation to a bottom-up model). Continuing Remark 2.1 we consider a bottom-up model with $m$ entities and associated default times $\tau_{1}, \ldots, \tau_{m}$. The loss process is

$$
L_{t}=\sum_{i=1}^{m} \mathbf{1}_{\left\{\tau_{i} \leq t\right\}} q_{i}
$$

where $q_{i}$ is the loss given default of entity $i$. Assume that $q_{i}$ are constant and $\tau_{i}$ has default intensity $\lambda_{i}$, that is

$$
\mathbf{1}_{\left\{\tau_{i} \leq t\right\}}-\int_{0}^{t} \mathbf{1}_{\left\{\tau_{i}>s\right\}} \lambda_{i}(s) d s
$$

is a martingale for $i=1, \ldots, m$. Then the compensator of $L$ is

$$
\nu^{L}(d t, d x)=F_{t}^{L}(d x) d t=\sum_{i=1}^{m} \lambda_{i}(t) \mathbf{1}_{\left\{\tau_{i}>t\right\}} \delta_{\left\{q_{i}\right\}}(d x) d t .
$$

For intuition consider i.i.d. exponentially distributed $\tau_{i}$ where the intensity parameter is $\lambda$ and $q_{i}=q$. Then

$$
F_{t}^{L}(d x)=\lambda \sum_{i=1}^{m} \mathbf{1}_{\left\{\tau_{i}>t\right\}} \delta_{\{q\}}(d x)=\lambda\left(m-q^{-1} L_{t}\right) \delta_{\{q\}}(d x) .
$$

Note that the compensator naturally depends on the number of defaults that have occurred already: as less and less entities remain in the pool, the intensity for a further loss decreases.

\section{AN AFFINE SPECIFICATION}

Affine processes are a powerful tool for yield curve modeling because they represent a rich class of processes, allowing for jumps and stochastic volatility, while still retaining a high degree of tractability. For examples see Cuchiero, Filipović, and Teichmann (2009), and Errais, Giesecke, and Goldberg (2010) for self-exciting affine processes. Duffie and Gârleanu (2001) is to our knowledge the first paper using affine jump-diffusions for modeling of stochastic intensities of single obligors in a dynamic bottom-up credit portfolio model. This section will illustrate how these processes can be used in our setup. Note that this is very different from the setting in Filipović, Overbeck, and Schmidt (2011); already Example 6.1 illustrates that Gaussian behavior of the spreads in a model with discrete tenor structure is possible, while in their setting this would generate arbitrage possibilities, see also 
Remark 5.3. Moreover, in our approach we are able to find an affine specification which includes contagion as we will show in the following.

For simplicity we discuss only the case of affine processes which are driven by a diffusion and a constant term structure as in Remark 4.1. Denote by $\mathcal{T}:=$ $\left\{T_{0}, \ldots, T_{n}\right\}$ the tenor structure and let $\mathcal{Z} \subset \mathbb{R}^{d}$ be some closed state space with nonempty interior. Consider a $d$-dimensional Brownian motion $W$ and let $\mu$ be defined on $\mathcal{Z}$ by

$$
\mu(z)=\mu_{0}+\sum_{i=1}^{d} \mu_{i} z_{i},
$$

for some vectors $\mu_{i} \in \mathbb{R}^{d}, i=0, \ldots, d$. Furthermore, we assume that $\sigma$ is defined on $\mathcal{Z}$ with values in $\mathbb{R}^{d \times d}$ such that

$$
\frac{1}{2} \sigma(z)^{\top} \sigma(z)=\nu_{0}+\sum_{i=1}^{d} \nu_{i} z_{i},
$$

for some matrices $\nu_{i} \in \mathbb{R}^{d \times d}, i=0, \ldots, d$. For any $z \in \mathcal{Z}$ we denote by $Z=Z^{z}$ the continuous, unique strong solution of

$$
d Z_{t}=\mu\left(Z_{t}\right) d t+\sigma\left(Z_{t}\right) d W_{t}, \quad Z_{0}=z .
$$

The class of models we consider are of the form

$$
\begin{aligned}
H\left(t, T_{k}, x\right)=\exp ( & A\left(t, T_{k}, x\right)+B\left(t, T_{k}, x\right)^{\top} Z_{t} \\
& \left.+\int_{0}^{t} \int_{I} c\left(s, T_{k}, x, L_{s-} ; y\right) \mu^{L}(d s, d y)+\int_{0}^{t} d\left(s, T_{k}, x, L_{s-}, Z_{s}\right) d s\right) .
\end{aligned}
$$

The first line is the part which is affine while the second part considers a contagion term which can have arbitrary dependence on $L$, but no dependence on $Z$. The term $d$ defines a drift which will compensate default and contagion risk. The assumptions on the functions $A, B, c$, and $d$ are as follows:

(B1) $A$ and $B$ satisfy the following system of Riccati equations:

$$
\begin{aligned}
-\partial_{t} A\left(t, T_{k}, x\right) & =B\left(t, T_{k}, x\right)^{\top} \mu_{0}+B\left(t, T_{k}, x\right)^{\top} 2 \nu_{0} \sum_{i=1}^{k} B\left(t, T_{i}, x\right) \\
& -B\left(t, T_{k}, x\right)^{\top} \nu_{0} B\left(t, T_{k}, x\right), \\
-\partial_{t} B\left(t, T_{k}, x\right)_{j} & =B\left(t, T_{k}, x\right)^{\top} \mu_{j}+B\left(t, T_{k}, x\right)^{\top} 2 \nu_{j} \sum_{i=1}^{k} B\left(t, T_{i}, x\right) \\
& -B\left(t, T_{k}, x\right)^{\top} \nu_{j} B\left(t, T_{k}, x\right),
\end{aligned}
$$

for $0 \leq t \leq T_{k}$.

(B2) The function $c: \mathbb{R}^{+} \times \mathcal{T} \times \mathcal{I} \times \mathcal{I} \times \mathcal{I}$ satisfies

$$
\sup _{t \leq T_{k}, x, l, y \in \mathcal{I}}\left|c\left(t, T_{k}, x, l ; y\right)\right|<\infty
$$

(B3) The compensator of the loss process satisfies $F_{t}^{L}(A)=m\left(t, L_{t-}, Z_{t}, A\right)$ for all $A \in \mathcal{B}(\mathcal{I})$ where $m(t, l, z, \cdot)$ is a $\sigma$-finite Borel measure for each $(t, l, z) \in$ 
$\mathbb{R}^{+} \times \mathcal{I} \times \mathcal{Z}$. Moreover $m$ is affine, i.e.

$$
m(t, l, z, \cdot)=m_{0}(t, l, \cdot)+\sum_{i=1}^{d} m_{i}(t, l, \cdot) z_{i}
$$

for some $m_{i}: \mathbb{R}^{+} \times \mathcal{I} \times \mathcal{B}(\mathcal{I}) \rightarrow \mathbb{R}^{+}, i=0, \ldots, d$.

(B4) The additional drift is affine, i.e.

$$
d\left(t, T_{k}, x, l, z\right)=d_{0}\left(t, T_{k}, x, l\right)+\sum_{i=1}^{d} d_{i}\left(t, T_{k}, x, l\right) z_{i}, \quad k=1, \ldots, n
$$

and

$$
\begin{aligned}
& d_{i}\left(t, T_{1}, x, l\right)=\int_{\mathcal{I}}\left(1-e^{c\left(t, T_{1}, x, l ; y\right)} \mathbf{1}_{\{y \leq x-l\}}\right) m_{i}(t, l, d y) \\
& d_{i}\left(t, T_{k}, x, l\right)=\int_{\mathcal{I}}\left(e^{\sum_{j=1}^{k-1} c\left(t, T_{j}, x, l ; y\right)}-e^{\sum_{j=1}^{k} c\left(t, T_{j}, x, l ; y\right)}\right) \mathbf{1}_{\{y \leq x-l\}} m_{i}(t, l, d y) \\
& \quad \text { for } i=0, \ldots, d \text { and } k=2, \ldots, n .
\end{aligned}
$$

Remark 7.1. Note that in (B3) we require $m(t, l, z, \cdot)$ not to be a signed measure. This implies restrictions on $m_{i}$ depending on the state space: if $\mathcal{Z}=\mathbb{R}^{d_{1}} \times\left(\mathbb{R}^{+}\right)^{d_{2}}$, with $d_{1}>0$ and $d=d_{1}+d_{2}$, then $m_{i}(t, l, \cdot)=0$ for $i=1, \ldots, d_{1}$ as otherwise there exist $z \in \mathcal{Z}$ such that

$$
m_{0}(t, l, A)+\sum_{i=1}^{d} m_{i}(t, l, A) z_{i}<0
$$

for some $l$ and $A$. This contradicts $F_{t}^{L}(A)=m\left(t, L_{t-}, Z_{t}, A\right) \geq 0$.

We assume that all functions which appear here are càdlàg in each variable.

The input parameters for the model are the coefficients $\mu_{i}, \nu_{i}$, as well as the contagion function $c$ and the Borel-measures $m_{i}, i=0, \ldots, d$. Note that we do not need to specify boundary conditions on the Riccati equations. They can be used to improve the fit on the initial term structure. The following proposition shows that the above conditions lead indeed to an arbitrage-free model.

Proposition 7.2. Assume (B1)-(B4). Then $\left(F\left(t, T_{k}, x\right)\right)_{0 \leq t \leq T_{k-1}}$ given by (16) with $H$ as in 24 are $\mathbb{Q}^{*}$-local martingales.

We start with a small lemma which is proved directly by applying Itô's formula.

Lemma 7.3. Consider $H$ as in (24) and assume that $A$ and $B$ are differentiable in $t$ with càdlàg derivatives. Then $H$ can be represented as in (11) with

$$
\begin{aligned}
a\left(t, T_{k}, x\right) & =\partial_{t} A\left(t, T_{k}, x\right)+\partial_{t} B\left(t, T_{k}, x\right)^{\top} Z_{t}+B\left(t, T_{k}, x\right)^{\top} \mu\left(Z_{t}\right) \\
& +d\left(t, T_{k}, x, L_{t-}, Z_{t}\right) \\
b\left(t, T_{k}, x\right) & =B\left(t, T_{k}, x\right)^{\top} \sigma\left(Z_{t}\right) \\
c\left(t, T_{k}, x ; y\right) & =c\left(t, T_{k}, x, L_{t-} ; y\right) .
\end{aligned}
$$

Proof of Proposition 7.2: Note that all assumptions of Theorem 5.2 are satisfied. In particular (A1) is trivially true since $F_{t}$ is 0 as a consequence of the continuity of $\left(Z_{t}\right)$. At the same time this allows to choose $\tilde{C}$ in $(\mathbf{A 1})$ equal to infinity and (A2) follows. 
Our aim is to show that the drift condition 19 is satisfied. In this regard, consider the case where $X$ is the $d$-dimensional Brownian motion $W$. We compute

$$
\begin{aligned}
& \sum_{i=1}^{k-1} a\left(t, T_{i}, x\right)+\frac{1}{2}\left\|\sum_{i=1}^{k-1} b\left(t, T_{i}, x\right)\right\|^{2} \\
& +\int_{\mathcal{I}}\left(e^{\sum_{i=1}^{k-1} c\left(t, T_{i}, x ; y\right)}-1\right) \mathbf{1}_{\left\{L_{t-}+y \leq x\right\}} F_{t}^{L}(d y)-\lambda(t, x) \\
& \quad=\sum_{i=1}^{k-1}\left(\partial_{t} A\left(t, T_{k}, x\right)+\partial_{t} B\left(t, T_{k}, x\right)^{\top} Z_{t}+B\left(t, T_{k}, x\right)^{\top} \mu\left(Z_{t}\right)\right) \\
& \quad+\frac{1}{2}\left\|\sum_{i=1}^{k-1} B\left(t, T_{i}, x\right)^{\top} \sigma\left(Z_{t}\right)\right\|^{2} \\
& \quad+\sum_{i=1}^{k-1} d\left(t, T_{i}, x, L_{t-}, Z_{t}\right) \\
& \quad+\int_{\mathcal{I}}\left(e^{\sum_{i=1}^{k-1} c\left(t, T_{i}, x, L_{t-} ; y\right)}-1\right) \mathbf{1}_{\left\{L_{t-}+y \leq x\right\}} m\left(t, L_{t-}, Z_{t}, d y\right)-\lambda(t, x) .
\end{aligned}
$$

Note that according to $(7) \lambda(t, x)=m\left(t, L_{t-}, Z_{t},\left(x-L_{t}, 1\right] \cap \mathcal{I}\right)$. Now we consider the equation above for all possible values $l \in \mathcal{I}$ of $L_{t}$ and $z \in \mathcal{Z}$ of $Z_{t}$. We have that $m(t, l, z,[0, x-l] \cap \mathcal{I})+m(t, l, z,(x-l, 1] \cap \mathcal{I})=m(t, l, z, \mathcal{I})$ and we obtain

$$
28]=\int_{\mathcal{I}} e^{\sum_{i=1}^{k-1} c\left(t, T_{i}, x, l ; y\right)} \mathbf{1}_{\{l+y \leq x\}} m(t, l, z, d y)-m(t, l, z, \mathcal{I}) .
$$

We set $z_{0} \equiv 1$ to simplify the notation. By (B4), we obtain

$$
\begin{aligned}
27]= & d\left(t, T_{1}, x, l, z\right)+\sum_{i=2}^{k-1} d\left(t, T_{i}, x, l, z\right) \\
= & \sum_{j=0}^{d} z_{j}\left(\int_{\mathcal{I}}\left(1-e^{c\left(t, T_{1}, x, l ; y\right)} \mathbf{1}_{\{y \leq x-l\}}\right) m_{j}(t, l, d y)\right. \\
& \left.\quad+\sum_{i=2}^{k-1} \int_{\mathcal{I}}\left(e^{\sum_{j^{\prime}=1}^{i-1} c\left(t, T_{j^{\prime}}, x, l ; y\right)}-e^{\sum_{j^{\prime}=1}^{i} c\left(t, T_{j^{\prime}}, x, l ; y\right)}\right) \mathbf{1}_{\{y \leq x-l\}} m_{j}(t, l, d y)\right) \\
= & \sum_{j=0}^{d} z_{j}\left(\int_{\mathcal{I}}\left(1-e^{\sum_{j^{\prime}=1}^{k-1} c\left(t, T_{j^{\prime}}, x, l ; y\right)} \mathbf{1}_{\{y \leq x-l\}}\right) m_{j}(t, l, d y)\right) \\
= & \int_{\mathcal{I}}\left(1-e^{\sum_{j^{\prime}=1}^{k-1} c\left(t, T_{j^{\prime}}, x, l ; y\right)} \mathbf{1}_{\{y \leq x-l\}}\right) m(t, l, z, d y) .
\end{aligned}
$$


Hence, 27$)+28)=0$. Our final step consists in proving that

$$
\begin{aligned}
0 & =\sum_{i=1}^{k-1}\left(\partial_{t} A\left(t, T_{i}, x\right)+\partial_{t} B\left(t, T_{i}, x\right)^{\top} z+B\left(t, T_{i}, x\right)^{\top} \mu(z)\right) \\
& +\frac{1}{2}\left\|\sum_{i=1}^{k-1} B\left(t, T_{i}, x\right)^{\top} \sigma(z)\right\|^{2} .
\end{aligned}
$$

As this equation is affine in $z$, i.e. of the form $\sum_{i=0}^{d} \alpha_{i} z_{i}$, it is sufficient to show that $\alpha_{i}=0$ for $i=0, \ldots, d$. First, we consider $\alpha_{0}$ and show that

$$
0=\sum_{i=1}^{k-1}\left(\partial_{t} A\left(t, T_{i}, x\right)+B\left(t, T_{i}, x\right)^{\top} \mu_{0}\right)+\sum_{i, j=1}^{k-1} B\left(t, T_{i}, x\right)^{\top} \nu_{0} B\left(t, T_{j}, x\right) .
$$

Note that 23) implies that $\nu_{j}$ is symmetric for any $j=1, \ldots, d$. Hence, by $(\mathbf{B 1})$,

$$
\begin{aligned}
0 & =\sum_{i=1}^{k-1}\left(\partial_{t} A\left(t, T_{i}, x\right)+B\left(t, T_{i}, x\right)^{\top} \mu_{0}\right) \\
& +\sum_{i=1}^{k-1} B\left(t, T_{i}, x\right)^{\top} \nu_{0} \sum_{j=1}^{i} B\left(t, T_{j}, x\right) \\
& +\sum_{i=1}^{k-1} B\left(t, T_{i}, x\right)^{\top} \nu_{0} \sum_{j=1}^{i} B\left(t, T_{j}, x\right) \\
& -\sum_{i=1}^{k-1} B\left(t, T_{i}, x\right)^{\top} \nu_{0} B\left(t, T_{i}, x\right)
\end{aligned}
$$

an this is exactly $(30)$. In a similar way, $(\mathbf{B 1})$ yields

$$
0=\sum_{i=1}^{k-1}\left(\partial_{t} B\left(t, T_{i}, x\right)_{j}+B\left(t, T_{i}, x\right)^{\top} \mu_{j}\right)+\sum_{i, l=1}^{k-1} B\left(t, T_{i}, x\right)^{\top} \nu_{j} B\left(t, T_{l}, x\right)
$$

for $j=1, \ldots, d$ such that 29 is proven. Summarizing, we obtain that the drift condition 190 holds and we conclude by Theorem 5.2

Remark 7.4. The previous proof shows that the coupled Riccati equations for $A$ and $B$ may be simplified by considering

$$
A^{k}(t, x):=\sum_{i=1}^{k} A\left(t, T_{i}, x\right), \quad B^{k}(t, x):=\sum_{i=1}^{k} B\left(t, T_{i}, x\right) .
$$

Then $(25)$ and 26 are equivalent to

$$
\begin{array}{r}
-\partial_{t} A^{k}(t, x)=B^{k}(t, x) \mu_{0}+B^{k}(t, x)^{\top} \nu_{0} B^{k}(t, x) \\
-\partial_{t} B^{k}(t, x)_{j}=B^{k}(t, x) \mu_{j}+B^{k}(t, x)^{\top} \nu_{j} B^{k}(t, x)
\end{array}
$$

for $k=1, \ldots, n$ and $j=1, \ldots, d$. Equations (31) and (32) are the classical Riccati equations for multivariate affine processes. In dimension $d=1$ the solutions are well-known, while in the general case efficient numerical schemes are available to compute $A^{k}$ and $B^{k}$. 
Up to now the modeling was quite general. In the following example we give a concrete one-dimensional affine specification which is much simpler. We will use a two-dimensional extension later on in the section on calibration.

Example 7.5. We choose a Feller square-root process as a driver: consider $d=1$ and $\mu_{0} \geq 0, \mu_{1} \in \mathbb{R}$ as well as $\nu_{1}=\sigma^{2} / 2$. Then

$$
d Z_{t}=\left(\mu_{0}+\mu_{1} Z_{t}\right) d t+\sigma \sqrt{Z_{t}} d W_{t},
$$

with $Z_{0}=z>0$. The Feller condition $2 \mu_{1}>\sigma^{2}$ ensures positivity of $Z$. In this case the Riccati equations (31) and (32) have explicit solutions, see for example Cuchiero, Filipović, and Teichmann (2009). The compensator of the loss process is specified via

$$
m(t, l, z, d y)=m_{0}+m_{1} p_{\alpha, \beta}(d y) z,
$$

where $p_{\alpha, \beta}$ is a $\operatorname{Beta}(\alpha, \beta)$-distribution. Finally, the contagion parameter is assumed to be a function of the loss process, i.e.

$$
c\left(t, T_{k}, x, l ; y\right)=c\left(T_{k}-t, y\right) .
$$

Choosing $c$ decreasing in $y$ guarantees that upward jumps in the loss process lead to downward jumps in the price process, and hence to upward jumps in the credit spreads. Computing the terms $d_{1}, \ldots, d_{k}$ by a simple numerical integration is the last step for specifying an arbitrage-free model.

\section{PRICING OF PORTFOlio CREDIT DERIVATIVES}

In this section we study the valuation of portfolio credit derivatives. In particular, we focus our attention on single tranche CDOs (STCDOs) and call options on STCDOs.

8.1. Single tranche CDO. The valuation of derivatives can often be facilitated by using appropriate defaultable forward measures. We illustrate this by considering a standard instrument for investment in a credit pool, a so-called single tranche CDO. A single tranche $C D O$ ( $\mathrm{STCDO})$ is specified by:

- a collection of future dates (tenor dates) $T_{1}<T_{2}<\cdots<T_{m}$,

- lower and upper detachment points $x_{1}<x_{2}$ in $[0,1]$

- a fixed spread $S$.

The STCDO offers premium in exchanges for payments at defaults: the premium leg (received by the investor) consists of a series of payments equal to

$$
S\left[\left(x_{2}-L_{T_{k}}\right)^{+}-\left(x_{1}-L_{T_{k}}\right)^{+}\right],
$$

received at $T_{k}, k=1, \ldots, m-1$. Letting

$$
f(x):=\left(x_{2}-x\right)^{+}-\left(x_{1}-x\right)^{+}=\int_{x_{1}}^{x_{2}} \mathbf{1}_{\{x \leq y\}} \mathrm{d} y,
$$

we have that $(33)=S f\left(L_{T_{k}}\right)$.

The default leg (paid by the investor) consists of a series of payments at times $T_{k+1}, k=1, \ldots, m-1$, given by

$$
f\left(L_{T_{k}}\right)-f\left(L_{T_{k+1}}\right) .
$$


This payment is nonzero only if $\Delta L_{t} \neq 0$ for some $t \in\left(T_{k}, T_{k+1}\right]$. In the literature alternative payment schemes can be found as well (see Filipović, Overbeck, and Schmidt (2011), for example). We have

$$
\text { (35) }=\int_{x_{1}}^{x_{2}}\left[\mathbf{1}_{\left\{L_{T_{k}} \leq y\right\}}-\mathbf{1}_{\left\{L_{T_{k+1}} \leq y\right\}}\right] \mathrm{d} y=\int_{x_{1}}^{x_{2}} \mathbf{1}_{\left\{L_{T_{k}} \leq y, L_{T_{k+1}}>y\right\}} \mathrm{d} y .
$$

Let us denote by $e\left(t, T_{k+1}, x\right)$ the value at time $t$ of a payment given by $\mathbf{1}_{\left\{L_{T_{k}} \leq x, L_{T_{k+1}}>x\right\}}$ at the tenor date $T_{k+1}$. To calculate $e\left(t, T_{k+1}, x\right)$, it is convenient to replace the measure $\mathbb{Q}_{T_{k+1}}$ by a new one. As already discussed, the market trades only financial instruments whose first tenor date is at least a full tenor period away. In this regard we introduce a time horizon $\delta<T_{1}$ and consider the forward prices on $[0, \delta]$. Applying Theorem 5.2 with respect to the tenor structure $\left\{\delta, T_{1}, \ldots, T_{m}\right\}$ yields an arbitrage-free construction of forward prices. Assume

(A5) The processes $\left(F\left(t, T_{k}, x\right)\right)_{0 \leq t \leq T_{k-1}}$, are true $\mathbb{Q}_{T_{k}}$-martingales for every $k=$ $2, \ldots, n$ and $x \in \mathcal{I}$. Moreover, $\left(F\left(t, T_{1}, x\right)\right)_{0 \leq t \leq \delta}$ is a true $\mathbb{Q}_{T_{1}}$-martingale.

Assumption (A5) allows us to switch to a measure under which the numeraire is given by the $\left(T_{k}, x\right)$-forward price. This is not an equivalent measure change, but it still yields a measure which is absolutely continuous with respect to the initial one. Similar measure changes have been introduced in Schönbucher (2000) and have been successfully applied to the pricing of credit risky securities, cf. Eberlein, Kluge, and Schönbucher (2006). Let $x \in[0,1]$ and $k \in\{1, \ldots, m-1\}$. We define the $\left(T_{k+1}, x\right)$ forward measure $\mathbb{Q}_{T_{k+1}, x}$ on $\left(\Omega, \mathcal{G}_{T_{k+1}}\right)$ by its Radon-Nikodym derivative

$$
\frac{\mathrm{d} \mathbb{Q}_{T_{k+1}, x}}{\mathrm{~d} \mathbb{Q}_{T_{k+1}}}:=\frac{F\left(T_{k}, T_{k+1}, x\right)}{\mathbb{E}_{\mathbb{Q}_{T_{k+1}}}\left[F\left(T_{k}, T_{k+1}, x\right)\right]}=\frac{F\left(T_{k}, T_{k+1}, x\right)}{F\left(0, T_{k+1}, x\right)},
$$

where the last equality follows under (A5). The corresponding density process is

$$
\left.\frac{\mathrm{d} \mathbb{Q}_{T_{k+1}, x}}{\mathrm{~d} \mathbb{Q}_{T_{k+1}}}\right|_{\mathcal{G}_{t}}=\frac{F\left(t, T_{k+1}, x\right)}{F\left(0, T_{k+1}, x\right)} .
$$

As already mentioned, $\mathbb{Q}_{T_{k+1}, x}$ is not equivalent to $\mathbb{Q}_{T_{k+1}}$ if $\mathbb{Q}_{T_{k+1}}\left(L_{T_{k}}>x\right)>0$.

Lemma 8.1. Assume (A5). Let $x \in \mathcal{I}$ and $k \in\{1, \ldots, m-1\}$. Then, for every $t \leq T_{k}$,

$$
e\left(t, T_{k+1}, x\right)=P\left(t, T_{k+1}, x\right) \mathbb{E}_{\mathbb{Q}_{T_{k+1}, x}}\left(\prod_{i=0}^{k} H\left(T_{i}, T_{i}, x\right)^{-1}-1 \mid \mathcal{G}_{t}\right) .
$$

Proof: The price at time $t$ of a contingent claim with payoff

$$
e\left(T_{k+1}, T_{k+1}, x\right)=\mathbf{1}_{\left\{L_{T_{k}} \leq x\right\}}-\mathbf{1}_{\left\{L_{T_{k+1}} \leq x\right\}}
$$

at $T_{k+1}$ equals

$$
e\left(t, T_{k+1}, x\right)=P\left(t, T_{k+1}\right) \mathbb{E}_{\mathbb{Q}_{T_{k+1}}}\left(\mathbf{1}_{\left\{L_{T_{k}} \leq x\right\}}-\mathbf{1}_{\left\{L_{T_{k+1}} \leq x\right\}} \mid \mathcal{G}_{t}\right) .
$$

Regarding the second term, observe that

$$
P\left(t, T_{k+1}\right) \mathbb{E}_{\mathbb{Q}_{T_{k+1}}}\left(\mathbf{1}_{\left\{L_{T_{k+1}} \leq x\right\}} \mid \mathcal{G}_{t}\right)=P\left(t, T_{k+1}, x\right)
$$


by (A5). For the first term we have

$$
\begin{aligned}
\mathbb{E}_{\mathbb{Q}_{T_{k+1}}}\left(\mathbf{1}_{\left\{L_{T_{k}} \leq x\right\}} \mid \mathcal{G}_{t}\right) & \\
= & \mathbb{E}_{\mathbb{Q}_{T_{k+1}}}\left(\mathbf{1}_{\left\{L_{T_{k}} \leq x\right\}}\left(\prod_{i=0}^{k} H\left(T_{k}, T_{i}, x\right)\right)\left(\prod_{i=0}^{k} H\left(T_{k}, T_{i}, x\right)\right)^{-1} \mid \mathcal{G}_{t}\right) \\
& =\mathbb{E}_{\mathbb{Q}_{T_{k+1}}}\left(F\left(T_{k}, T_{k+1}, x\right) \prod_{i=0}^{k} H\left(T_{i}, T_{i}, x\right)^{-1} \mid \mathcal{G}_{t}\right),
\end{aligned}
$$

which follows from (16) and $H\left(t, T_{i}, x\right)=H\left(T_{i}, T_{i}, x\right)$, for $t \geq T_{i}$. Changing to the measure $\mathbb{Q}_{T_{k+1}, x}$ yields

$$
\mathbb{E}_{\mathbb{Q}_{T_{k+1}}}\left(\mathbf{1}_{\left\{L_{T_{k}} \leq x\right\}} \mid \mathcal{G}_{t}\right)=F\left(t, T_{k+1}, x\right) \mathbb{E}_{\mathbb{Q}_{T_{k+1}, x}}\left(\prod_{i=0}^{k} H\left(T_{i}, T_{i}, x\right)^{-1} \mid \mathcal{G}_{t}\right) .
$$

Therefore,

$$
\begin{aligned}
e\left(t, T_{k+1}, x\right) & =P\left(t, T_{k+1}\right) \frac{P\left(t, T_{k+1}, x\right)}{P\left(t, T_{k+1}\right)} \mathbb{E}_{\mathbb{Q}_{T_{k+1}, x}}\left(\prod_{i=0}^{k} H\left(T_{i}, T_{i}, x\right)^{-1} \mid \mathcal{G}_{t}\right) \\
& -P\left(t, T_{k+1}, x\right) \\
& =P\left(t, T_{k+1}, x\right) \mathbb{E}_{\mathbb{Q}_{T_{k+1}, x}}\left(\prod_{i=0}^{k} H\left(T_{i}, T_{i}, x\right)^{-1}-1 \mid \mathcal{G}_{t}\right)
\end{aligned}
$$

and the lemma is proved.

Proposition 8.2. Assume (A5). Then the value of the STCDO at any time $t \in[0, \delta]$ is

$$
\pi^{S T C D O}(t, S)=\int_{x_{1}}^{x_{2}}\left(S \sum_{k=1}^{m-1} P\left(t, T_{k}, y\right)-\sum_{k=1}^{m-1} e\left(t, T_{k+1}, y\right)\right) \mathrm{d} y .
$$

Recall that the premium $S f\left(L_{T_{k}}\right)$ is paid at times $T_{1}, \ldots, T_{m-1}$, whereas the default payments are due at time points $T_{2}, \ldots, T_{m}$.

Proof: The value of the premium leg at time $t$ equals

$$
\begin{aligned}
\sum_{k=1}^{m-1} P\left(t, T_{k}\right) \mathbb{E}_{\mathbb{Q}_{T_{k}}}\left(S f\left(L_{T_{k}}\right) \mid \mathcal{G}_{t}\right) & =\sum_{k=1}^{m-1} S P\left(t, T_{k}\right) \int_{x_{1}}^{x_{2}} \mathbb{E}_{\mathbb{Q}_{T_{k}}}\left(\mathbf{1}_{\left\{L_{T_{k}} \leq y\right\}} \mid \mathcal{G}_{t}\right) \mathrm{d} y \\
& =S \sum_{k=1}^{m-1} \int_{x_{1}}^{x_{2}} P\left(t, T_{k}, y\right) \mathrm{d} y
\end{aligned}
$$


where we have used (37). On the other side, the default payment at time $T_{k+1}$ is given by $f\left(L_{T_{k}}\right)-f\left(L_{T_{k+1}}\right)$. Its value at time $t$ is equal to

$$
\begin{aligned}
P\left(t, T_{k+1}\right) & \mathbb{E}_{\mathbb{Q}_{T_{k+1}}}\left(f\left(L_{T_{k}}\right)-f\left(L_{T_{k+1}}\right) \mid \mathcal{G}_{t}\right) \\
= & P\left(t, T_{k+1}\right) \mathbb{E}_{\mathbb{Q}_{T_{k+1}}}\left(\int_{x_{1}}^{x_{2}} \mathbf{1}_{\left\{L_{T_{k}} \leq y, L_{T_{k+1}}>y\right\}} \mathrm{d} y \mid \mathcal{G}_{t}\right) \\
= & \int_{x_{1}}^{x_{2}} P\left(t, T_{k+1}\right) \mathbb{E}_{\mathbb{Q}_{T_{k+1}}}\left(\mathbf{1}_{\left\{L_{T_{k}} \leq y, L_{T_{k+1}}>y\right\}} \mid \mathcal{G}_{t}\right) \mathrm{d} y \\
= & \int_{x_{1}}^{x_{2}} e\left(t, T_{k+1}, y\right) \mathrm{d} y .
\end{aligned}
$$

Hence, the value of the default leg at time $t$ is given by

$$
\sum_{k=1}^{m-1} \int_{x_{1}}^{x_{2}} e\left(t, T_{k+1}, y\right) \mathrm{d} y .
$$

Finally, the value of the STCDO is the difference of these two values and thus we obtain (38).

The STCDO spread $S_{t}^{*}$ at time $t$ is the spread which makes the value of the STCDO equal to zero, i.e. one has to solve $\pi^{S T C D O}(t, S)=0$. The previous proposition yields

$$
S_{t}^{*}=\frac{\int_{x_{1}}^{x_{2}} \sum_{k=1}^{m-1} e\left(t, T_{k+1}, y\right) \mathrm{d} y}{\int_{x_{1}}^{x_{2}} \sum_{k=1}^{m-1} P\left(t, T_{k}, y\right) \mathrm{d} y} .
$$

Corollary 8.3. Assume (A5) and assume that the default-free bond prices $P\left(\cdot, T_{k}\right)$ and the loss process $L$ are conditionally independent given $\mathcal{G}_{t}$, for all $k \in\{1, \ldots, n\}$ and $t \in[0, \delta]$. Then

$$
e\left(t, T_{k+1}, x\right)=P\left(t, T_{k+1}\right) F\left(t, T_{k}, x\right)-P\left(t, T_{k+1}, x\right) .
$$

Proof: Conditional independence of $P\left(\cdot, T_{k}\right)$ and $L$ implies

$$
\mathbb{E}_{\mathbb{Q}_{T_{k+1}}}\left(\mathbf{1}_{\left\{L_{T_{k}} \leq x\right\}} \mid \mathcal{G}_{t}\right)=\mathbb{E}_{\mathbb{Q}_{T_{k}}}\left(\mathbf{1}_{\left\{L_{T_{k}} \leq x\right\}} \mid \mathcal{G}_{t}\right),
$$

since $\left.\frac{d \mathbb{Q}_{T_{k}}}{\mathrm{~d}_{T_{k+1}}}\right|_{\mathcal{G}_{t}}=\frac{P\left(0, T_{k+1}\right)}{P\left(0, T_{k}\right)} \frac{P\left(t, T_{k}\right)}{P\left(t, T_{k+1}\right)}$ is the density process for this change of measure (cf. equation 12). Then

$$
\mathbb{E}_{\mathbb{Q}_{T_{k+1}}}\left(\mathbf{1}_{\left\{L_{T_{k}} \leq x\right\}} \mid \mathcal{G}_{t}\right)=\frac{P\left(t, T_{k}, x\right)}{P\left(t, T_{k}\right)}=F\left(t, T_{k}, x\right)
$$

and we obtain from (36)

$$
e\left(t, T_{k+1}, x\right)=P\left(t, T_{k+1}\right) F\left(t, T_{k}, x\right)-P\left(t, T_{k+1}, x\right) .
$$


Corollary 8.4. Under the assumptions of Corollary 8.3, the price at time $t \in[0, \delta]$ of the STCDO is given by

$$
\pi^{S T C D O}(t, S)=\int_{x_{1}}^{x_{2}}\left(\sum_{k=1}^{m} c_{k} P\left(t, T_{k}\right) F\left(t, T_{k}, y\right)-\sum_{k=1}^{m-1} P\left(t, T_{k+1}\right) F\left(t, T_{k}, y\right)\right) \mathrm{d} y,
$$

where $c_{1}=S, c_{k}=1+S$, for $2 \leq k \leq m-1$, and $c_{m}=1$. The STCDO spread $S_{t}^{*}$ at time $t \in[0, \delta]$ is equal to

$$
S_{t}^{*}=\frac{\sum_{k=1}^{m-1} \int_{x_{1}}^{x_{2}} P\left(t, T_{k+1}\right)\left(F\left(t, T_{k}, y\right)-F\left(t, T_{k+1}, y\right)\right) \mathrm{d} y}{\sum_{k=1}^{m-1} \int_{x_{1}}^{x_{2}} P\left(t, T_{k}\right) F\left(t, T_{k}, y\right) \mathrm{d} y} .
$$

Proof: Follows by inserting (41) into (38) and (40).

Remark 8.5. Corollary 8.4 shows that under conditional independence of the default-free bond prices and the loss process, the STCDO spreads are given in terms of the initial term structure of the default-free bond prices and the $\left(T_{k}, x\right)$-forward prices. This allows one to extract $\left(T_{k}, x\right)$-forward prices from market data.

8.2. Options on a STCDO. Consider a STCDO as defined in the previous subsection. Let us study an option which gives the right to enter into such a contract at time $T_{1}$ at a pre-specified spread $S$. This is equivalent to a European call on the STCDO with payoff

$$
\left(\pi^{S T C D O}\left(T_{1}, S\right)\right)^{+}
$$

at $T_{1}$. Assume that (A5) holds. The value of the European call at time $t \in[0, \delta]$ is given by the expectation under the forward measure $\mathbb{Q}_{T_{1}}$ :

$$
\begin{aligned}
\pi^{\text {call }}(t, S) & =P\left(t, T_{1}\right) \mathbb{E}_{\mathbb{Q}_{T_{1}}}\left(\left(\pi^{S T C D O}\left(T_{1}, S\right)\right)^{+} \mid \mathcal{G}_{t}\right) \\
& =P\left(t, T_{1}\right) \mathbb{E}_{\mathbb{Q}_{T_{1}}}\left(\left(\int_{x_{1}}^{x_{2}}\left(S \sum_{k=1}^{m-1} P\left(T_{1}, T_{k}, y\right)-\sum_{k=1}^{m-1} e\left(T_{1}, T_{k+1}, y\right)\right) \mathrm{d} y\right)^{+} \mid \mathcal{G}_{t}\right),
\end{aligned}
$$

since by 38 ,

$$
\pi^{S T C D O}\left(T_{1}, S\right)=\int_{x_{1}}^{x_{2}}\left(S \sum_{k=1}^{m-1} P\left(T_{1}, T_{k}, y\right)-\sum_{k=1}^{m-1} e\left(T_{1}, T_{k+1}, y\right)\right) \mathrm{d} y .
$$

Assuming for simplicity that $P\left(t, T_{k}\right)=1$, for all $T_{k}$ and $t \leq T_{k}$, which implies the conditional independence which is assumed in Corollaries 8.3 and 8.4 , we obtain

$$
\pi^{\text {call }}(t, S)=\mathbb{E}_{\mathbb{Q}^{*}}\left(\left(\int_{x_{1}}^{x_{2}} \sum_{k=1}^{m} d_{k} F\left(T_{1}, T_{k}, y\right) \mathrm{d} y\right)^{+} \mid \mathcal{G}_{t}\right),
$$

where $d_{1}=S-1, d_{k}=S$, for $2 \leq k \leq m-1$, and $d_{m}=1$, which follows from (42). Note that the measure $\mathbb{Q}_{T_{1}}$ coincides with the terminal forward measure $\mathbb{Q}^{*}=\mathbb{Q}_{T_{n}}$, 
cf. Remark 4.1, Recall that

$$
\begin{aligned}
F\left(T_{1}, T_{k}, y\right) & =F\left(0, T_{k}, y\right) \exp \left(\sum_{i=1}^{k-1} \int_{0}^{T_{1}} a\left(t, T_{i}, y\right) \mathrm{d} t\right. \\
& \left.+\sum_{i=1}^{k-1} \int_{0}^{T_{1}} b\left(t, T_{i}, y\right) d X_{t}+\sum_{i=1}^{k-1} \int_{0}^{T_{1}} \int_{\mathcal{I}} c\left(t, T_{i}, y ; z\right) \mu^{L}(d t, d z)\right) \mathbf{1}_{\left\{L_{T_{1}} \leq y\right\}},
\end{aligned}
$$

for $k \geq 2$ and $F\left(T_{1}, T_{1}, y\right)=F\left(0, T_{1}, y\right) \mathbf{1}_{\left\{L_{T_{1}} \leq y\right\}}$. We further assume $F\left(0, T_{i}, y\right)$, $a\left(t, T_{i}, y\right), b\left(t, T_{i}, y\right)$ and $c\left(t, T_{i}, y ; z\right)$ are constant in $y$ between $x_{1}$ and $x_{2}$. For simplicity we denote $a\left(t, T_{i}, y\right)=a\left(t, T_{i}, x_{1}\right)$ by $a\left(t, T_{i}\right)$ and similarly for the other quantities. Then we have

$$
\begin{aligned}
\int_{x_{1}}^{x_{2}} \sum_{k=1}^{m} d_{k} F\left(T_{1}, T_{k}, y\right) \mathrm{d} y & =\sum_{k=1}^{m} d_{k} F\left(0, T_{k}\right) \exp \left(\sum_{i=1}^{k-1} \int_{0}^{T_{1}} a\left(t, T_{i}\right) \mathrm{d} t+\sum_{i=1}^{k-1} \int_{0}^{T_{1}} b\left(t, T_{i}\right) d X_{t}\right. \\
& \left.+\sum_{i=1}^{k-1} \int_{0}^{T_{1}} \int_{\mathcal{I}} c\left(t, T_{i} ; z\right) \mu^{L}(d t, d z)\right) \int_{x_{1}}^{x_{2}} \mathbf{1}_{\left\{L_{T_{1}} \leq y\right\}} \mathrm{d} y \\
& =f\left(L_{T_{1}}\right) \sum_{k=1}^{m} d_{k} F\left(0, T_{k}\right) \exp \left(\sum_{i=1}^{k-1} \int_{0}^{T_{1}} a\left(t, T_{i}\right) \mathrm{d} t\right. \\
& \left.+\sum_{i=1}^{k-1} \int_{0}^{T_{1}} b\left(t, T_{i}\right) d X_{t}+\sum_{i=1}^{k-1} \int_{0}^{T_{1}} \int_{\mathcal{I}} c\left(t, T_{i} ; z\right) \mu^{L}(d t, d z)\right),
\end{aligned}
$$

for $f$ defined in (34). Note that $f: \mathcal{I} \rightarrow \mathcal{I}$, and so $f\left(L_{T_{1}}\right) \geq 0$. Thus, the value of the option at time $t$ is given by

$$
\begin{aligned}
\pi^{\text {call }}(t, S) & =\mathbb{E}_{\mathbb{Q}^{*}}\left(f ( L _ { T _ { 1 } } ) \left(\tilde{d}_{1}+\sum_{k=2}^{m} \tilde{d}_{k} \exp \left(\sum_{i=1}^{k-1} \int_{0}^{T_{1}} a\left(t, T_{i}\right) \mathrm{d} t\right.\right.\right. \\
& \left.\left.\left.+\sum_{i=1}^{k-1} \int_{0}^{T_{1}} b\left(t, T_{i}\right) d X_{t}+\sum_{i=1}^{k-1} \int_{0}^{T_{1}} \int_{\mathcal{I}} c\left(t, T_{i} ; z\right) \mu^{L}(d t, d z)\right)\right)^{+} \mid \mathcal{G}_{t}\right),
\end{aligned}
$$

where $\tilde{d}_{k}=d_{k} F\left(0, T_{k}\right)$, for $1 \leq k \leq m$. Assume now that $L$ and $X$ are conditionally independent given $\mathcal{G}_{t}$. Therefore, if $c=0$ and $a\left(\cdot, T_{i}\right)$ and $b\left(\cdot, T_{i}\right)$ are conditionally independent of $L$ given $\mathcal{G}_{t}$ for all $T_{i}$, this expression simplifies further to

$$
\begin{aligned}
\pi^{\text {call }}(t, S)=\mathbb{E}_{\mathbb{Q}^{*}} & \left(f\left(L_{T_{1}}\right) \mid \mathcal{G}_{t}\right) \mathbb{E}_{\mathbb{Q}^{*}}\left(\left(\tilde{d}_{1}+\sum_{k=2}^{m} \tilde{d}_{k} \exp \left(\sum_{i=1}^{k-1} \int_{0}^{T_{1}} a\left(t, T_{i}\right) \mathrm{d} t\right.\right.\right. \\
& \left.\left.\left.+\sum_{i=1}^{k-1} \int_{0}^{T_{1}} b\left(t, T_{i}\right) d X_{t}\right)\right)^{+} \mid \mathcal{G}_{t}\right),
\end{aligned}
$$


where

$$
\begin{aligned}
\mathbb{E}_{\mathbb{Q}^{*}}\left(f\left(L_{T_{1}}\right) \mid \mathcal{G}_{t}\right) & =\mathbb{E}_{\mathbb{Q}^{*}}\left(\left(x_{2}-x_{1}\right) \mathbf{1}_{\left\{L_{T_{1}} \leq x_{1}\right\}}+\left(x_{2}-L_{T_{1}}\right) \mathbf{1}_{\left\{x_{1}<L_{T_{1}} \leq x_{2}\right\}} \mid \mathcal{G}_{t}\right) \\
& =x_{2} \mathbb{Q}^{*}\left(L_{T_{1}} \leq x_{2} \mid \mathcal{G}_{t}\right)-x_{1} \mathbb{Q}^{*}\left(L_{T_{1}} \leq x_{1} \mid \mathcal{G}_{t}\right) \\
& -\mathbb{E}_{\mathbb{Q}^{*}}\left(L_{T_{1}} \mathbf{1}_{\left\{x_{1}<L_{T_{1}} \leq x_{2}\right\}} \mid \mathcal{G}_{t}\right) .
\end{aligned}
$$

As far as the second factor in $\pi^{\text {call }}(t, S)$ is concerned, it is similar to the expressions that appear in valuation formulas for swaptions in term structure models without defaults. It can be computed using Fourier transform techniques under appropriate technical assumptions; cf. Eberlein and Kluge (2006) and Keller-Ressel, Papapantoleon, and Teichmann (2011). In particular, we refer to Eberlein, Glau, and Papapantoleon (2010) and Eberlein (2012) for Fourier transform methods in a general semimartingale setting. For the affine specification given in Section 7 , this approach may be simplified further.

\section{Calibration}

In this section we give a calibration exercise with a two-factor affine diffusion which on one side shows the flexibility of our framework in a simple specification and further illustrates the implementation of the model. For the calibration, we use the affine model from Section 7 and implement an extended Kalman filter as suggested in Eksi and Filipović (2012). In contrast to typical calibration approaches we do not only fit to data of single days but to the data of a period of two and a half years, namely from February 2008 to August 2010. The model is able to provide a surprisingly good fit across the different tranches and maturities as we shall illustrate.

9.1. The dataset. The calibration is performed on data from the iTraxx Europe index, more specifically it consists of implied zero-coupon spreads of the iTraxx Europe ${ }^{1}$. In the market there are STCDOs on the iTraxx Europe with detachment points $\left\{x_{1}, \ldots, x_{J}\right\}=\{0,0.03,0.06,0.09,0.12,0.22,1\}$. The zero-coupon spreads are the quoted spreads of the STCDOs, in our notation given by

$$
R(t, \tau, j):=-\frac{1}{\tau} \log \left(\frac{1}{x_{j+1}-x_{j}} \int_{x_{j}}^{x_{j+1}} F(t, t+\tau, x) d x\right),
$$

where $\tau$ denotes time to maturity. In the data we have $\tau \in\{3,5,7,10\}$. In the model we consider later the case where $F(t, T, x)$ is constant in the intervals $\left[x_{j}, x_{j+1}\right)$ and then

$$
-\tau \cdot R(t, \tau, j)=\log P\left(t, t+\tau, x_{j}\right)-\log P(t, t+\tau)
$$

as $F(t, T, x)=P(t, T, x) P(t, T)^{-1}$ by definition. Therefore, the rate $R$ indeed refers to a spread above the risk-free rate.

The realized index spreads are shown in Figure 1. With the beginning of the credit crisis, volatility, as well as the credit spreads, jumped to very high levels stabilizing thereafter. In the first quarter of 2010 a new increase due to the European debt crises can easily be spotted. Figure 2 shows the evolution of the tranche spreads for different maturities and tranches. The spread curves follow a similar pattern. Consequently, it is plausible to capture the dynamics with a low number of factors.

\footnotetext{
${ }^{1}$ We thank Dr. Peter Schaller for providing us the data.
} 


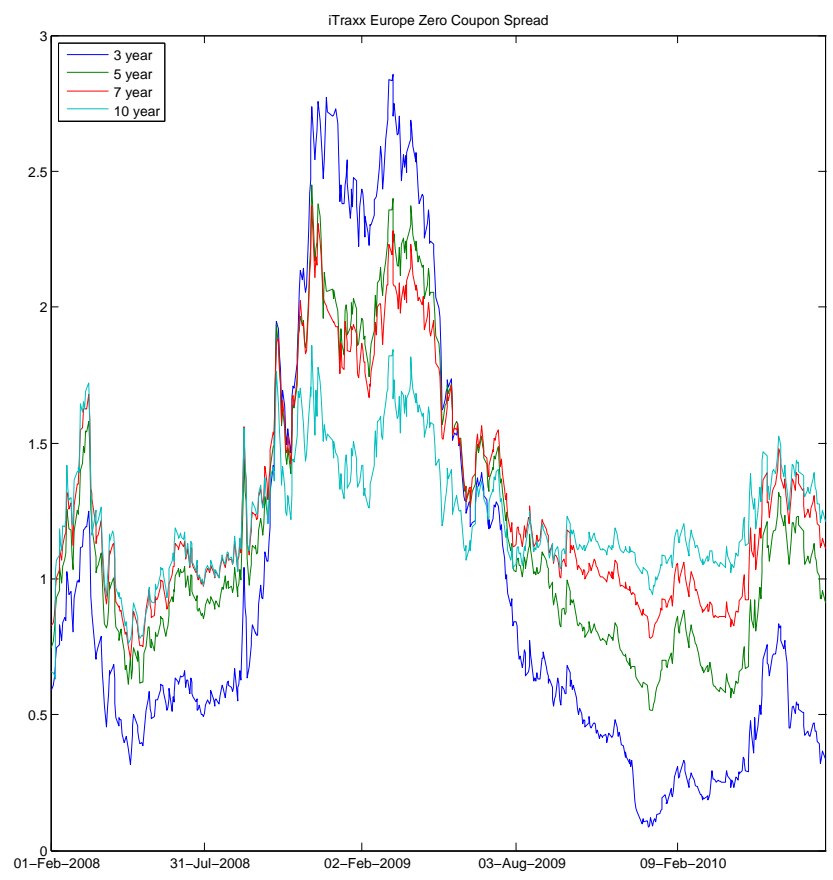

FiguRE 1. The iTraxx Europe zero-coupon index spread for the period February 2008 to August 2010. The different graphs refer to the time to maturity of $3,5,7$ and 10 years.

It is important to mention that in the observation period defaults did not occur in the underlying pool.

9.2. Model specification. Our aim is to calibrate a simple two-factor affine diffusion model to the whole data set using Kalman filtering. To this end, we specify the model under the physical probability measure $\mathbb{P}$. Prices of traded products are computed under the risk-neutral measure $\mathbb{Q}$ which we obtain by a change of measure where the affine structure is kept.

A principal component analysis reveals that two factors already explain $88.30 \%$ of the realized variance; see Eksi and Filipović (2012). We therefore consider a twodimensional affine process $Z=\left(Z^{1}, Z^{2}\right)^{\mid} \in \mathbb{R}^{+} \times \mathbb{R}^{+}=: \mathcal{Z}$ satisfying

$$
\begin{aligned}
& d Z_{t}^{1}=\kappa^{1}\left(Z_{t}^{2}-Z_{t}^{1}\right) d t+\sigma^{1} \sqrt{Z_{t}^{1}} d W_{t}^{1} \\
& d Z_{t}^{2}=\kappa^{2}\left(\theta^{2}-Z_{t}^{2}\right) d t+\sigma^{2} \sqrt{Z_{t}^{2}} d W_{t}^{2},
\end{aligned}
$$

and $Z_{0}=\left(z_{1}, z_{2}\right) \in \mathcal{Z}$. Here $\kappa^{1}, \kappa^{2}, \theta^{2}, \sigma^{1}, \sigma^{2}$ are positive constants and $W^{1}$ and $W^{2}$ are independent standard Brownian motions. The factor $Z^{2}$ is the stochastic mean reversion level of $Z^{1}$. 

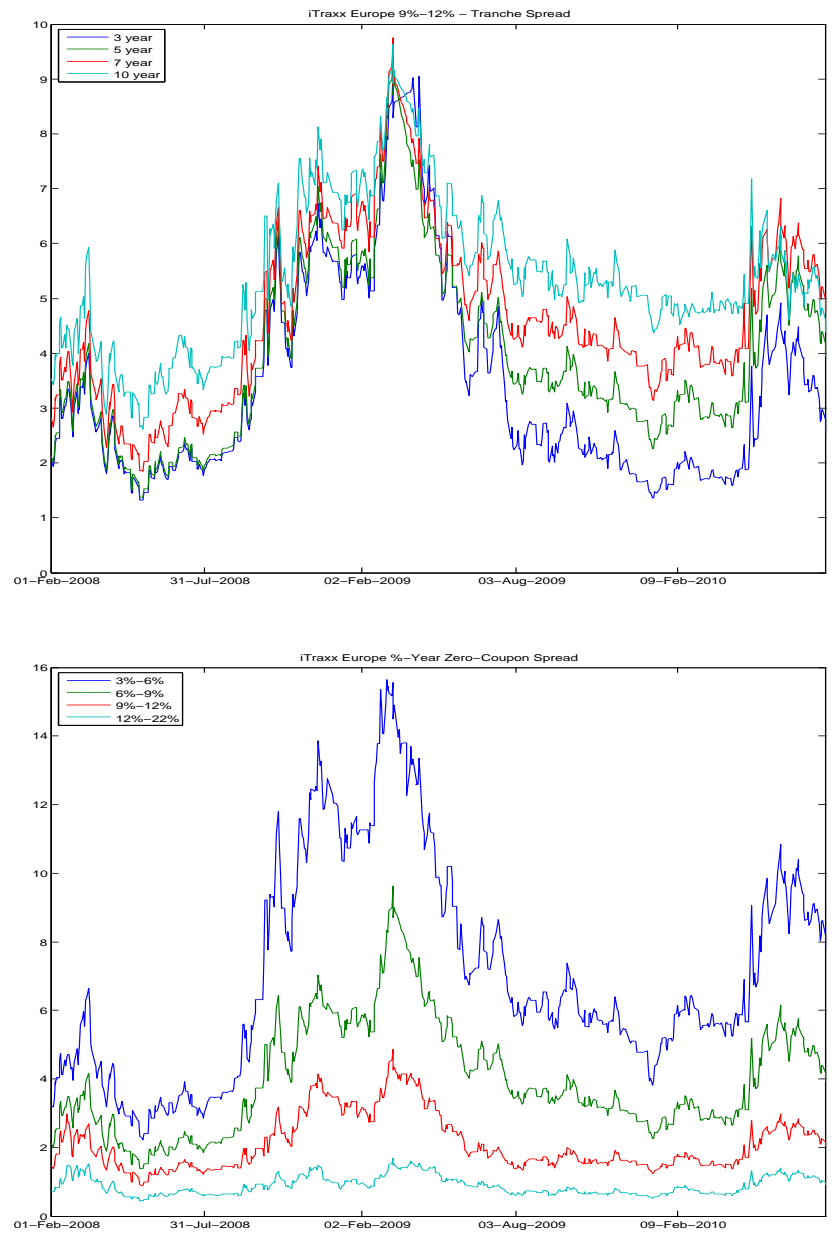

FiguRE 2. The upper graph shows the iTraxx Europe 9\%-12\% tranche spread from February 2008 to August 2010 for different maturities. The lower graph illustrates the iTraxx Europe tranche spreads from February 2008 to August 2010 for a fixed maturity of five years.

For the measure change we specify the market prices of risk by

$$
\lambda_{t}^{i}=\frac{\lambda^{i} \sqrt{Z_{t}^{i}}}{\sigma^{i}}, \quad i=1,2
$$

with constants $\lambda^{1}, \lambda^{2} \in \mathbb{R}$. Using Girsanov's theorem, we change to an equivalent measure $\mathbb{Q}$ where $\tilde{W}_{t}^{i}=W_{t}^{i}+\int_{0}^{t} \lambda_{s}^{i} d s, i=1,2$ are independent standard Brownian motions. Then, under $\mathbb{Q}, Z$ is again affine and satisfies the following dynamics; see Cheridito, Filipović, and Kimmel (2010):

$$
\begin{aligned}
& d Z_{t}^{1}=\left(\kappa^{1}+\lambda^{1}\right)\left(\frac{\kappa^{1}}{\kappa^{1}+\lambda^{1}} Z_{t}^{2}-Z_{t}^{1}\right) d t+\sigma^{1} \sqrt{Z_{t}^{1}} d \tilde{W}_{t}^{1} \\
& d Z_{t}^{2}=\left(\kappa^{2}+\lambda^{2}\right)\left(\frac{\kappa^{2}}{\kappa^{2}+\lambda^{2}} \theta^{2}-Z_{t}^{2}\right) d t+\sigma^{2} \sqrt{Z_{t}^{2}} d \tilde{W}_{t}^{2}
\end{aligned}
$$


Hence, $Z$ is an affine process under $\mathbb{Q}$ and we may apply the results from Section 7 .

For a complete specification of the model we need to specify the compensator of the loss process $L$ and the contagion parameter $c$. According to our setup we assume that $m$ depends in an affine way on $Z$ and we assume that it is only driven by $Z^{1}$, i.e.

$$
m(t, l, z, d y)=m_{0}(t, l, d y)+m_{1}(t, l, d y) z_{1} .
$$

We choose the jump distribution from the beta family, more precisely

$$
m(t, l, z, d y)=\frac{1}{B\left(a_{1}, b_{1}\right)} y^{a_{1}-1}(1-y)^{b_{1}-1} d y+\frac{z_{1}}{B\left(a_{2}, b_{2}\right)} y^{a_{2}-1}(1-y)^{b_{2}-1} d y,
$$

where all coefficients are positive. Finally we specify the contagion parameter and assume that

$$
c\left(t, T_{k}, x, L_{t-} ; y\right)=c y\left(T_{k}-t\right) .
$$

We consider $H$ specified as in (24) together with (25) and (26) and it follows from Proposition 7.2 that this is an arbitrage-free model.

9.3. The calibration procedure. For the estimation of the (unobserved) variables $Z$ from the observed STCDO prices we use an extended Kalman filter following Eksi and Filipović (2012). Furthermore we make the following two assumptions: first, we assume that tranche spreads are piecewise constant between the detachment points, that is

$$
H\left(t, T_{k}, x\right)=H\left(t, T_{k}, x_{i+1}\right), \quad \text { for } x \in\left[x_{i}, x_{i+1}\right) .
$$

Second, we assume that observed prices are given by model implied prices with additive noise. More formally, we assume that at observation times $0=t_{0}, t_{1}, t_{2}, \ldots$

$$
\begin{aligned}
R\left(t_{k}, \tau, j\right) & =-\frac{1}{\tau} \log \left(\frac{1}{x_{j+1}-x_{j}} \int_{x_{j}}^{x_{j+1}} F\left(t_{k}, t+\tau, x\right) d x\right)+\varepsilon(k, \tau, j+1) \\
& =: \alpha\left(\tau, x_{j+1}\right)-\frac{1}{\tau} \beta\left(\tau, x_{j+1}\right) Z_{t_{k}}-c L_{t_{k}}+\varepsilon(k, \tau, j+1) .
\end{aligned}
$$

Note that with $H$ also $F$ is affine. Moreover, as $A$ and $B$ are piecewise constant, the terms $\alpha$ and $\beta$ are straightforward to compute, see Gehmlich, Grbac, and Schmidt (2013) for detailed computations. The measurement error consists of independent and normally distributed random variables, where the variance of the measurement errors may differ across the observed tranches: $\varepsilon(k, \tau, j+1) \sim \mathcal{N}\left(0, \sigma_{j+1}\right)$.

We approximate the conditional distribution of $Z_{t_{k}}$ given $Z_{t_{k-1}}$ by a normal distribution where the first and the second moments are matched. This is in line with a quasi-maximum-likelihood approach and simplifies the computations considerably. The moments of the affine diffusion $Z$ can be computed using the Kolmogorov backward equation; see Proposition 3.1 in Eksi and Filipović (2012). This enables us to apply the extended Kalman filter algorithm to obtain a calibration to the full data set. The details of this approach and the extension to more factors can be found in Gehmlich, Grbac, and Schmidt (2013).

Remark 9.1. As an alternative to the filtering approach which we favor here one could also use nonlinear least squares to fit the model to data. Such an approach is pursued in Longstaff and Rajan (2008), notably on a quite different model. They fit the unknown parameter vector $\theta$, as well as the unobserved factor process $Z$, to the data by minimizing the sum of squared distances between the observed prices 
and the model prices computed with parameter $\theta$ and the factor process $Z_{1}, \ldots, Z_{T}$ taking values $z_{1}, \ldots, z_{T}$. Applying this procedure to market data of the CDX NA IG for the period from October 2003 to October 2005 they fit a three-factor model. A comparison to the filtering approach reveals on one side, that nonlinear least squares give only access to the parameters under the risk-neutral measure. On the other side, with the filtering approach one gets additional regularity on the estimated factor process in comparison to nonlinear least squares. In this regard, it is surprising that the model considered here is able to provide an excellent fit to a longer and more turbulent time series with two factors only. For details we refer to the calibration results in the following section.

9.4. Calibration results. The extended Kalman filter allows a calibration to the full dataset from February 2008 to August 2010. On one side, the Kalman filter provides an estimation of the hidden state process $Z$ and on the other side, maximizing the quasi-likelihood function given the estimated values of $Z$, gives the estimator of the parameter vector. Table 1 shows the estimated values.

\begin{tabular}{cccccccccccc}
\hline$\lambda^{1}$ & $\lambda^{2}$ & $\kappa^{1}$ & $\kappa^{2}$ & $\theta^{2}$ & $\sigma^{1}$ & $\sigma^{2}$ & $c$ & $a_{1}$ & $b_{1}$ & $a_{2}$ & $b_{2}$ \\
\hline-0.0780 & -2.5472 & 1.5722 & 1.8569 & 0.4720 & 0.7305 & 0.1739 & -0.0571 & 0.6797 & 5.1597 & 0.2492 & 22.26 \\
\hline
\end{tabular}

TABLE 1. Estimated parameter values.

It turns out, that the jump distribution in $m_{1}$ is quite close to an exponential distribution, as $a_{2}$ is small. However, $a_{1}$ contributes significantly to the fit of the model. The contagion parameter $c$ is negative, as expected - an occurring loss, i.e. an upward jump in the loss process leads to a downward jump in the $(T, x)$-bond prices by a downward jump in $H$.

Based upon the estimated parameter values and the filtered factor process we regenerate the data. In Figures 3 and 4 we plot estimated vs. observed values. For brevity, the longest maturity which shows a similar behavior is left aside. The graphs can be used for the diagnosis of the model fit. It is remarkable that the two-factor model is able to provide an excellent fit across all tranches and over the whole data period. This underlines the stability of the approach which leads to improved hedging performance, as shown in Eksi and Filipović (2012). They obtain a similar fit with a two-factor affine model when incorporating additionally a catastrophic component. As pointed out, a two-factor model with zero catastrophic component is not able to provide a good fit to the super-senior tranche. In our approach, the additional freedom obtained by considering a discrete tenor structure allows to incorporate a contagion term which improves the fit substantially. Compare in particular Figure 4. 

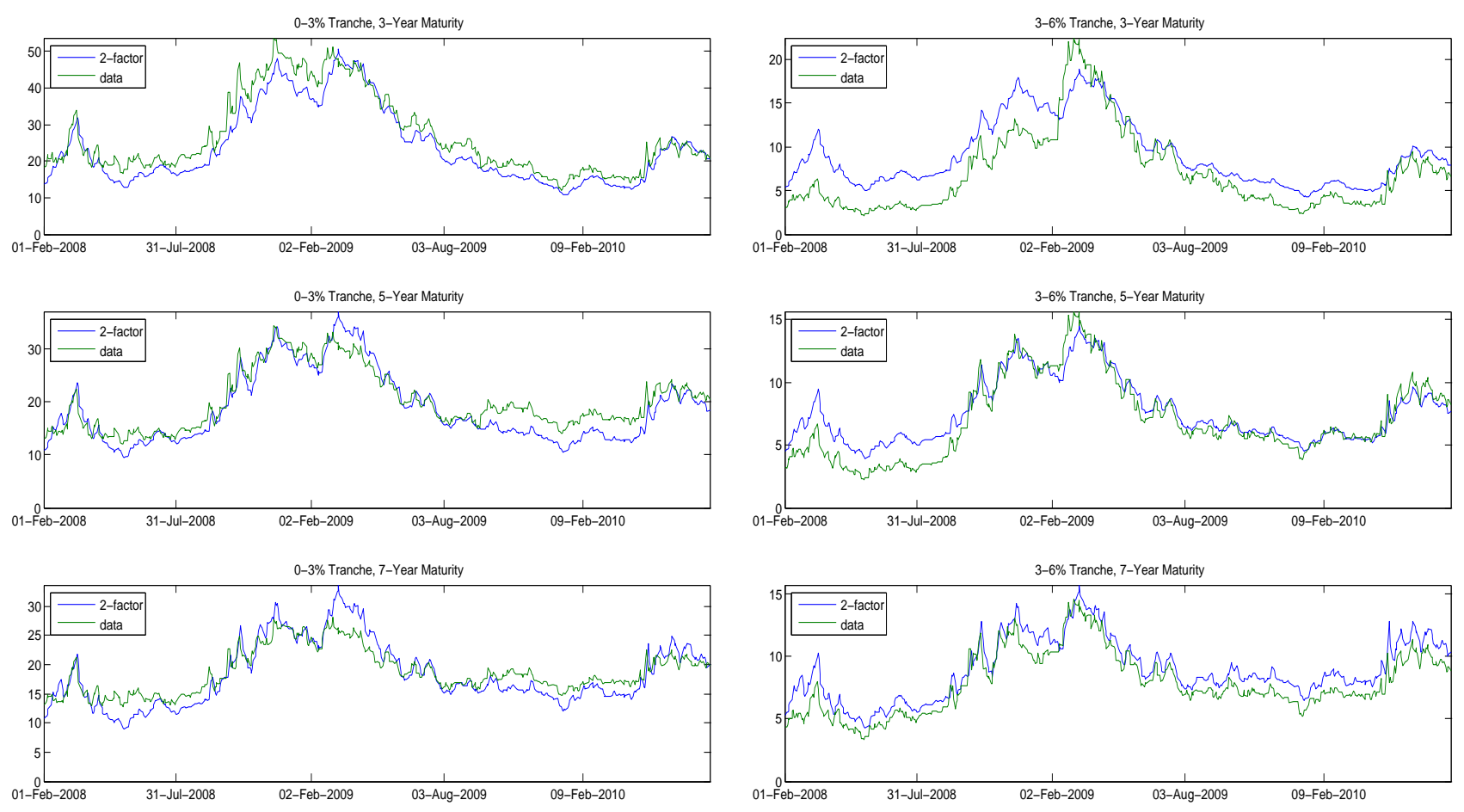

0\%-3\% Tranche

$3 \%-6 \%$ Tranche
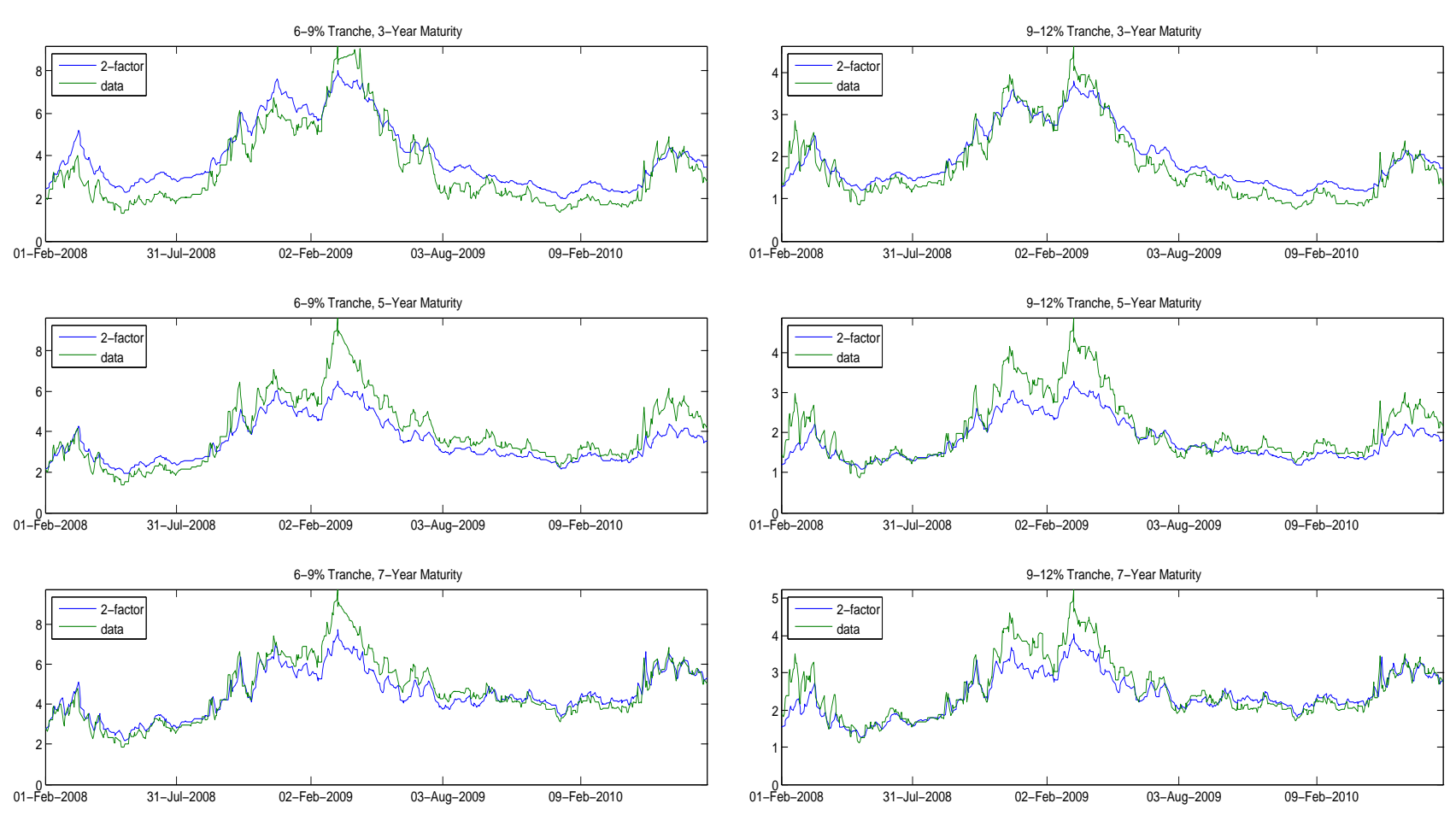

6\%-9\% Tranche

9\%-12\% Tranche

Figure 3. Estimated and realized data - part 1. 

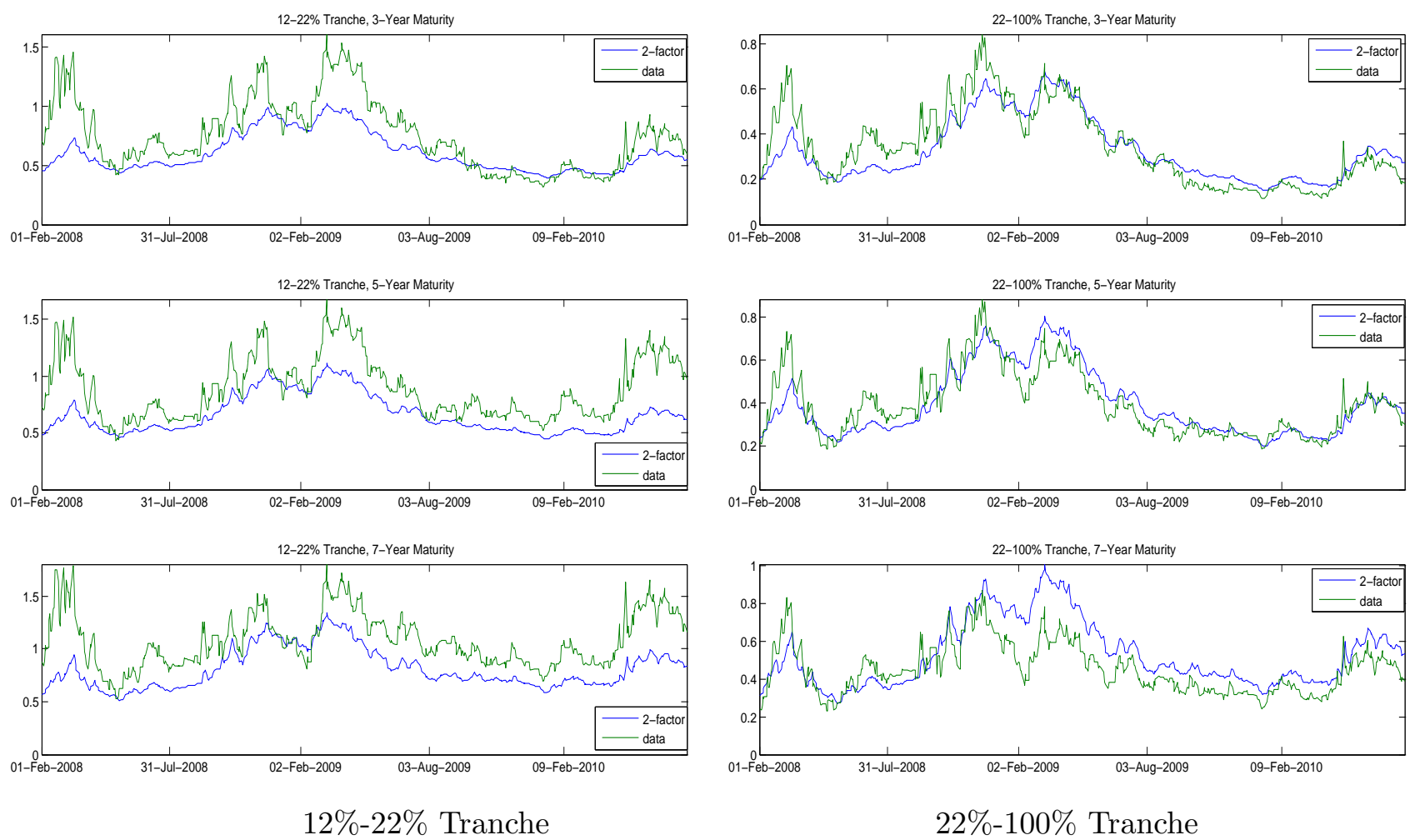

12\%-22\% Tranche

22\%-100\% Tranche

Figure 4. Estimated and realized data - part 2.

\section{REFERENCES}

Arnsdorf, M. and I. Halperin (2008). BSLP: Markovian bivariate spread-loss model for portfolio credit derivatives. Journal of Computational Finance 12, 77-107.

Bennani, N. and D. Dahan (2004). An extended market model for credit derivatives. Stochastic Finance Autumn School \& International Conference, available at http://nordine.bennani.free.fr/_private/ecmm.pdf.

Bielecki, T. R., S. Crépey, and M. Jeanblanc (2010). Up and down credit risk. Quantitative Finance 10, 1137-1151.

Carpentier, P. (2009). A market model on the iTraxx. Risk 22(11), 84-89.

Cheridito, P., D. Filipović, and R. L. Kimmel (2010). A note on the Dai-Singleton canonical representation of affine term structure models. Mathematical Finance 20(3), 509-519.

Cont, R., R. Deguest, and Y. H. Kan (2010). Default intensities implied by CDO spreads: inversion formula and model calibration. SIAM Journal on Financial Mathematics 1, 555-585.

Cont, R. and Y. H. Kan (2011). Dynamic hedging of portfolio credit derivatives. SIAM Journal on Financial Mathematics 2, 112-140. 
Cont, R. and A. Minca (2013). Recovering portfolio default intensities implied by CDO quotes. Mathematical Finance 23, 94-121.

Cuchiero, C., D. Filipović, and J. Teichmann (2009). Affine models. In R. Cont (Ed.), Encyclopedia of Quantitative Finance, Wiley.

Duffie, D. and N. Gârleanu (2001). Risk and valuation of collateralized debt obligations. Financial Analysts Journal 57, 41-59.

Eberlein, E. (2012). Fourier based valuation methods in mathematical finance. In Financial Engineering for Energy Asset Management and Hedging in Commodity Markets; Proceedings from the special thematic year at the Wolfgang Pauli Institute, Vienna, Editor: F.E. Benth. Springer (forthcoming).

Eberlein, E., K. Glau, and A. Papapantoleon (2010). Analysis of Fourier transform valuation formulas and applications. Applied Mathematical Finance 17, 211240.

Eberlein, E. and W. Kluge (2006). Exact pricing formulae for caps and swaptions in a Lévy term structure model. Journal of Computational Finance 9(2), 99125.

Eberlein, E., W. Kluge, and P. J. Schönbucher (2006). The Lévy Libor model with default risk. Journal of Credit Risk 2(2), 3-42.

Eberlein, E. and N. Koval (2006). A cross-currency Lévy market model. Quantitative Finance 6(6), 465-480.

Eberlein, E. and F. Özkan (2005). The Lévy Libor model. Finance and Stochastics 9, 327-348.

Ehlers, P. and P. Schönbucher (2006). Pricing interest rate-sensitive credit portfolio derivatives. Swiss Finance Institute Research Paper No. 06-39.

Ehlers, P. and P. Schönbucher (2009). Background filtrations and canonical loss processes for top-down models of portfolio credit risk. Finance and Stochastics 13, 79-103.

Eksi, Z. and D. Filipović (2012). A dynamic affine factor model for the pricing of CDOs. Working paper.

Errais, E., K. Giesecke, and L. R. Goldberg (2010). Affine point processes and portfolio credit risk. SIAM Journal on Financial Mathematics 1, 642-665.

Filipović, D., L. Overbeck, and T. Schmidt (2011). Dynamic CDO term structure modeling. Mathematical Finance 21, 53-71.

Gehmlich, F., Z. Grbac, and T. Schmidt (2013). Pricing and calibration in market models. In Credit Securitisations and Derivatives, Editors: H. Scheule and D. Rösch. Wiley (forthcoming).

Jacod, J. and A. N. Shiryaev (2003). Limit Theorems for Stochastic Processes (2nd ed.). Springer.

Keller-Ressel, M., A. Papapantoleon, and J. Teichmann (2011). The affine LIBOR models. Mathematical Finance. (forthcoming).

Lipton, A. and A. Rennie (Eds.) (2011). The Oxford Handbook of Credit Derivatives. Oxford University Press, London.

Longstaff, F. and A. Rajan (2008). An empirical analysis of collateralized debt obligations. The Journal of Finance 63, 529-563.

Sato, K.-I. (1999). Lévy Processes and Infinitely Divisible Distributions. Cambridge University Press. 
Schmidt, T. and J. Zabczyk (2012). CDO term structure modelling with Lévy processes and the relation to market models. International Journal of Theoretical and Applied Finance 15(1).

Schönbucher, P. J. (2000). A Libor market model with default risk. Working paper, University of Bonn.

Schönbucher, P. J. (2005). Portfolio losses and the term structure of loss transition rates: a new methodology for the pricing of portfolio credit derivatives. Working paper.

Sidenius, J., V. Piterbarg, and L. Andersen (2008). A new framework for dynamic credit portfolio loss modelling. International Journal of Theoretical and Applied Finance 11, 163-197.

Ernst Eberlein, University of Freiburg, Department of Mathematical Stochastics, Eckerstr. 1, 79104 Freiburg, Germany

E-mail address: eberlein@stochastik.uni-freiburg.de

Zorana Grbac, Institute of Mathematics, TU Berlin, Str. des 17. Juni 136, 10623 Berlin, Germany

E-mail address: grbac@math.tu-berlin.de

Thorsten Schmidt, Chemnitz University of Technology, Reichenhainer Str. 41, 09126 Chemnitz, Germany

E-mail address: thorsten.schmidt@mathematik.tu-chemnitz.de 